Neutron fibres: a possible application of neutron optics

This content has been downloaded from IOPscience. Please scroll down to see the full text. 1984 J. Phys. D: Appl. Phys. 17475

(http://iopscience.iop.org/0022-3727/17/3/007)

View the table of contents for this issue, or go to the journal homepage for more

Download details:

IP Address: 147.96.14.15

This content was downloaded on 04/02/2014 at 12:52

Please note that terms and conditions apply. 


\title{
Neutron fibres: a possible application of neutron optics $\dagger$
}

\author{
R F Alvarez-Estrada and M L Calvo $\$$ \\ $\div$ Departemento de Física Teórica, Facultad de Ciencias Físicas. Universidad \\ Complutense, Madrid-3, Spain \\ $\$$ Departemento de Optica y Estructura de la Materia, Facultad de Ciencias Físicas, \\ Universidad Complutense. Madrid-3. Spain
}

Received 7 April 1983, in final form 8 July 1983

\begin{abstract}
In this paper we study the possible confined propagation of slow (thermal) neutrons along (non-hollow) solid fibres made of certain elements (Ti, Mn....) such that the amplitude for the low-energy nuclear scattering of neutrons on them be negative. Geometrical optics and quasiclassical descriptions, procedures for confining the neutrons. and some applications to radiotherapy are outlined. The confined propagation modes for slow neutrons along infinite homogeneous circular fibres without and with nuclear absorption are analysed. Some estimates of the spectral density in the neutron flux and its attenuation are outlined. In order to assess lattice periodicity effects, the potential seen by a neutron inside an infinite periodic solid fibre and its confined propagation are discussed in detail. Here we find wide allowed and small forbidden bands for the neutron energy along the periodic fibre: well inside the allowed bands the neutron propagation modes reduce, as a first approximation. to those for the homogeneous fibre. Lattice vibration effects and the penetration, confinement and eventual scattering of neutrons by a homogeneous fibre are also analysed.
\end{abstract}

\section{Introduction}

Several typical optical effects (reflection, refraction, interference, diffraction and transmission) for slow neutrons with associated de Broglie wavelengths about $1 \AA$ have been analysed theoretically and established experimentally (see Hughes 1954, Bacon 1962. Sears 1982, Klein and Werner 1983).

Moreover, hollow neutron guides, which allow for the transport of neutron beams inside empty tubes having suitable walls ( $\mathrm{Ni}, \mathrm{Cu}$, glass . . ) by multiple internal reflections of the neutrons on the latter, have been studied (see Christ and Springer 1962, MaierLeibnitz and Springer 1963, Hofmeyr and Isebeck 1974, Carlile et al 1979, Klein and Werner 1983, Marx 1971, Jacrot 1970, Schaerpf and Eichler. 1973, Windsor 1981 and references therein).

In this paper, we shall study the possible existence of confined propagation modes for slow neutrons along cylindrical samples of certain specific materials (Ti, Mn, . . ). Our conjectures, if they can be supported by experiment, would contribute to establish another analogy between slow neutrons and light, which, as is well known, does have confined propagation modes along glass-like dielectric waveguides (optical fibres) under certain physical conditions.

$\doteqdot$ This paper is dedicated to Professor A Durán Miranda, Head of the Optics Department in the Universidad Complutense at the time of his retirement. In particular, we would like to remember his important contributions to the study of the nocturnal myopia and his persistent support and help, along many years and from various standpoints, to the development of optics and other different research areas in Spain 
Moreover, the possible practical disposal of confined neutron beams inside solid waveguides or fibres having a small cross-section could have several applications: for instance, the investigation of solid materials, slow neutron radiotherapy (connecting the hollow neutron guide to the patient), neutron-induced reactions, ... We are proposing that non-hollow solid waveguides, or fibres made up of certain elements (or alloys thereof) may also be useful, in addition to (and presumably combined with) the known hollow neutron guides.

This paper presents the following results. In $\S 2$, we discuss, in simple terms, the possibility of confining neutrons, the restricted class of materials for which such a confinement could occur and, briefly, some possible applications of the resulting neutron fibres.

In $\S 3$, we analyse the propagation modes for a neutron along an idealised homogeneous cylindrical fibre having infinite length and circular cross-section, disregarding nuclear absorption and also lattice periodicity and vibration effects. Some features of the propagation modes in a magnetised fibre are also discussed briefly.

In $\S 4$, we consider nuclear absorption effects on the neutron propagation modes: specifically, the imaginary parts of their propagation constants, which characterise the attenuation along the fibre are determined. Some estimates of the spectral density of the neutron flux and of its attenuation are also outlined.

The analysis in $\S \S 2,3$ and 4 leaves open the questions of whether lattice periodicity and vibration effects could be important in neutron fibres. Section 5 and appendix 1 are devoted to providing some answers.

In $\S 5$, we study the propagation modes in an infinite periodic fibre (without nuclear absorption). Here, the potential seen by a neutron is taken as that superposition of Fermi pseudopotentials which is strictly periodic along the fibre axis and corresponds to a rigid lattice, in close analogy to studies on neutron diffraction by crystals. We show the existence of (wide) allowed and (rather small) forbidden bands for the neutron energy along the fibre axis: well inside an allowed band (far from its edges), the neutron propagation modes in the periodic fibre reduce, as a first approximation, to those for the homogeneous one.

In appendix 1 , we consider a neutron inside an infinite three-dimensional vibrating crystal of any element having potential applications as a neutron fibre ( $\mathrm{Ti}, \mathrm{Mn}, \ldots$. .). A variational argument allows to justify the replacement of the neutron-phonon interaction by the same temperature-dependent potential (including the Debye-Waller factor) that is used in neutron diffraction studies. Then some numerical estimates show that the latter potential can be replaced, as a first approximation at ordinary temperatures $(\simeq 300 \mathrm{~K})$, by the standard sum of Fermi pseudopotentials that is used in $\S 5$ as a starting point.

In appendix 2 , we deal with the following problem. Given an arbitrary wave-function for a free neutron approaching the fibre, we outline a theoretical construction of that part of the wave-function which corresponds to a superposition of propagation modes along the fibre, thereby separating it from a remainder which describes pure scattering (but never propagation modes). The formal constructions of that remainder and of the scattered wave produced by it are also outlined.

\section{Neutron confinement: general theory}

\subsection{General aspects and discussion of confining materials}

The basis for the study of optical phenomena for neutrons is the following approximate 
Schrödinger wave equation, which determines its propagation through given material media and vacuum:

$$
\left(-\frac{\hbar^{2}}{2 m} \Delta+V(\boldsymbol{x})\right) \psi(\boldsymbol{x})=E \psi(\boldsymbol{x})
$$

Here, $\psi(\boldsymbol{x}), m$ and $E$ are the time-independent coherent (two-component) wave-function, the mass and the total energy for a non-relativistic neutron, respectively. On the other hand, $V(\boldsymbol{x})$ is the approximate potential on the neutron due to a given material medium, while $V(x)=0$ for $x$ in vacuum.

For the sake of simplicity we shall consider first neutron propagation in an homogeneous medium, omitting the following effects: (i) neutron magnetic interactions, (ii) crystal periodicity and lattice vibrations, (iii) those arising from the fact that the medium is not infinite along all directions, (iv) neutron absorption by the nuclei. We shall consider these effects later. Then, inside the material medium we can approximate (Hughes 1954 , Lax 1951, Sears 1982)

$$
V(\boldsymbol{x})=\frac{2 \pi \hbar^{2} \rho b}{m}
$$

where $\rho$ is the number of nuclei per unit volume $\left(\mathrm{cm}^{3}\right)$, and $b$ is the real average bound coherent amplitude for the purely nuclear scattering of the neutron by a nucleus in the medium (at very low energy). For simplicity we have not written the unit $2 \times 2$ matrix, I, explicitly in the right-hand side of equation (2.1.2).

One could also take into account the so-called local field correction by replacing $b$ by $b c$, where $c$ is the material constant which describes it (see Lax 1952, Ekstein 1953, Sears 1982 and references therein). However, due to the relative smallness of such an effect $(|c-1| \ll 1$; see Sears (1982) for estimates), we shall disregard it and set $c=1$ everywhere.

The relative index of refraction, $n$, for neutrons with energy $E$ and wave-vector $K\left(E=\hbar^{2} K^{2} / 2 m\right)$ is given by

$$
n^{2}=1-\frac{4 \pi \rho b}{\boldsymbol{K}^{2}}
$$

(see Hughes 1954, ch 1, Bacon 1962, Sears 1982).

As it is well known, the existence of confined propagation modes of light inside suitable glass-like dielectric waveguides (optical fibres), in which the refractive index exceeds unity, has been analysed theoretically and demonstrated experimentally: the main physical requirement for that confinement is total internal reflection of light inside the waveguide, under the condition that the incidence angle exceeds the critical one (Marcuse 1972, Olshansky 1979).

As an attempt to extend the optical analogies between light and slow neutrons even further, we suggest that propagation modes for neutrons inside cylindrical samples of certain homogeneous materials could, in principle, exist, provided that the neutron refractive index $n$ exceeds unity or, equivalently, that the potential $V(x)$ seen by the neutron is attractive. There is no vacuum region inside such a solid waveguide, which is not hollow.

Both conditions are fulfilled if the nuclear scattering amplitude $b$ (eventually averaged over different isotopes) is negative. In table 1 (the data in which are taken from Koester et al 1981), we collect the rather reduced set of elements and isotopes having negative nuclear scattering amplitudes, and the corresponding values for the latter. For older compilations, see Shull and Wollan (1951) and Bacon (1962, pp 31-2). 
Table 1. Nuclear scattering amplitude $b$ (Koester et al 1981).

\begin{tabular}{llllllll}
\hline & \multicolumn{7}{c}{ Element } \\
& $\mathrm{H}$ & $\mathrm{Li}$ & $\mathrm{Ti}$ & $\mathrm{V}$ & $\mathrm{Mn}$ & ${ }^{62} \mathrm{Ni}$ & ${ }^{152} \mathrm{Sm}$ \\
\hline$b\left(10^{-12} \mathrm{~cm}\right)$ & -0.374 & -0.203 & -0.337 & -0.0385 & -0.373 & -0.87 & -0.5 \\
\hline
\end{tabular}

Table 1 shows that the largest value of $-b$ occurs for ${ }^{62} \mathrm{Ni}$. Unfortunately, only $3.7 \%$ of the total amount of $\mathrm{Ni}$ occurring naturally corresponds to ${ }^{62} \mathrm{Ni}$ and the $b$ value of $\mathrm{Ni}$. averaged over isotopes, turns out to be 1.03 (see Shull and Wollan 1951). In order to construct a pure $\mathrm{Ni}$ fibre confining neutrons, it would be necessary first to enrich its content of ${ }^{62} \mathrm{Ni}$ considerably.

With regard to the practical construction of a neutron fibre or solid waveguide, the following points should be considered.

2.1.1. Solid waveguides made up by a pure element. Among the available elements, titanium appears as a quite appropriate material, due to (i) its natural abundance (9th most abundant element), (ii) pure samples of it can be prepared in a laboratory, for instance, by heating $\mathrm{TiCl}_{4}$ (with $\mathrm{Na}$ ) (neutron diffraction studies on pure $\mathrm{Ti}$, to determine its associated nuclear scattering amplitude, were reported in Shull and Wollan (1951)), (iii) it has interesting mechanical, chemical and physical properties, and (iv) the attenuation of a neutron beam due to nuclear absorption along a $\mathrm{Ti}$ solid waveguide will be less than (or equal to) that for any other element listed in table 1 . This last will follow immediately from the values of the linear absorption coefficient in table 2 (see $\$ 4$ ). Manganese could also be interesting: it can also be isolated in a laboratory. Regarding neutron absorption by these nuclei, see the discussion in $\S 4$.

2.1.2. Solid waveguides made of alloys. One could, in principle, consider alloys containing some of the suitable elements belonging from table 1, the overall average nuclear scattering amplitude being still negative so that they could operate as neutron fibres. An 'isotopic' alloy of $62 \% \mathrm{Ti}$ and $38 \% \mathrm{Zr}$ (random substitutional solid solution) has already been made (Sidhu et al 1956). The effect of the negative nuclear scattering amplitude of Ti cancels that arising from the positive amplitude $\left(b=+0.62 \times 10^{-12} \mathrm{~cm}\right)$ of $\mathrm{Zr}$, and the absence of Bragg peaks which follows from such a cancellation was established experimentally by those authors.

Some previous works by other authors have considered possible peculiarities in the behaviour of neutrons in certain cases. Thus, the possible storage of cold neutrons was discussed in Zel'dovich (1959) from a standpoint and under conditions which are different from those considered in this paper (see also Steyerl 1977). On the other hand, a time delay (amounting to several seconds) of a neutron beam which interacted with a suitable sample of LiF was detected experimentally by Grant and Cobble (1969). Such an experiment motivated some theoretical discussion by Kagan (1970) "concerning a bound neutron in matter": here the bound state energy of such a neutron was estimated to be $10^{-7} \mathrm{eV}$ (see also the comments in Dederichs (1972)). Regarding the possibility of unlimited residence periods for neutrons in a crystal (such as a single perfect Si crystal), which could be exploited as 'crystal-stored neutrons', and for further interesting comments regarding the analogies between neutrons in crystals, radiation in waveguides and electrons in conducting crystals, see Shull et al (1980), and Slater (1958) respectively. 


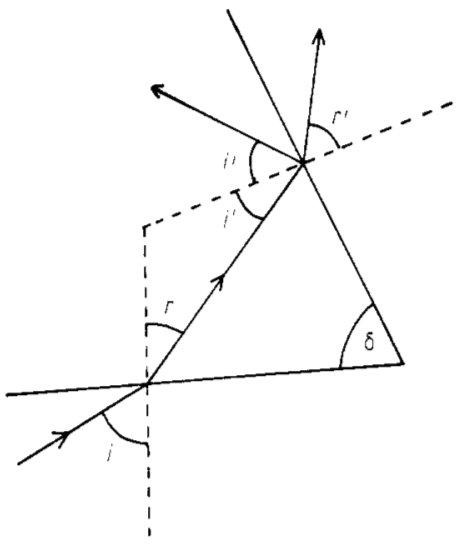

(a)

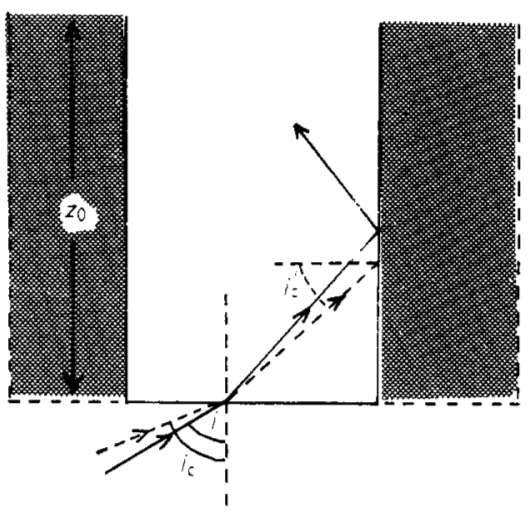

(b)

Figure 1. Refractions and reflections of a neutron in a fibre (or prism) with $n>1$. (a) General case; (b) total internal reflection and confined propagation for $\delta \leqslant \pi / 2, i<i_{\mathrm{c}}$.

\subsection{Neutron confinement by total internal reflection: some possible arrangements}

We consider the incidence of a neutron beam upon the entrance surface of a fibre (or one face of a prism) made of any of the elements listed in table 1, or an alloy thereof, so that the corresponding (averaged) nuclear amplitude be negative. We shall outline a simple geometric-optics treatment of the successive refractions and reflections suffered by an isolated neutron in this beam, as indicated in figure $1(a)$. We assume for the time being that the waveguide or prism is in a vacuum. Let $i$ and $r$ be the incidence and refraction angles at the entrance surface and $i^{\prime}, r^{\prime}$ those at the second surface, which forms an angle $\delta(\leqslant \delta / 2)$ with the first one (see figure $1(a)$ ). One has

$$
\sin i=n \sin r \quad n \sin i^{\prime}=\sin r^{\prime} \quad r+i^{\prime}=\delta
$$

$n(>1)$ being given by equation (2.1.3). Total internal reflection occurs at the second surface only if

$$
\begin{aligned}
& \sin r_{\mathrm{c}}^{\prime}=1 \quad \sin i_{\mathrm{c}}^{\prime}=n^{-1} \\
& \sin i_{\mathrm{c}}=\left(n^{2}-1\right)^{1 / 2} \sin \delta-\cos \delta .
\end{aligned}
$$

In particular, for a cylindrical solid waveguide with $\delta=\pi / 2$ (see figure $1(b)$, where the shaded regions represent the vacuum) the neutron will propagate confined inside the waveguide for any incidence angle $i<i_{\mathrm{c}}$.

For $\delta=\pi / 2$ and by using equations (2.1.3) and (2.2.1) for the physical values of $b$, $\rho$ for the elements listed in table 1 and for thermal neutrons with incoming energy $E$ outside the waveguide, one finds that the acceptance angle $i_{\mathrm{c}}$ is rather small, so that

$$
i_{\mathrm{c}} \simeq \frac{|4 \pi \rho(-b)|^{1 / 2} \hbar}{(2 m E)^{1 / 2}} .
$$

For instance, for $\mathrm{Ti}$, with $\rho \simeq 5.6 \times 10^{22}$ nuclei $\mathrm{cm}^{-3}$ and a thermal neutron energy $E \simeq 0.02 \mathrm{eV}$, one finds $i_{\mathrm{c}} \sim 1.57 \times 10^{-3} \mathrm{rad} \sim 5.39^{\prime}$.

Then, for a given waveguide length $z_{0}$, the smaller the size of its cross-section, the larger the confinement effect will be. Consider a cylindrical fibre with $z_{0}=10 \mathrm{~cm}$ and circular cross-section $\Omega$ of radius $R$, and the incidence of a neutron beam at the 


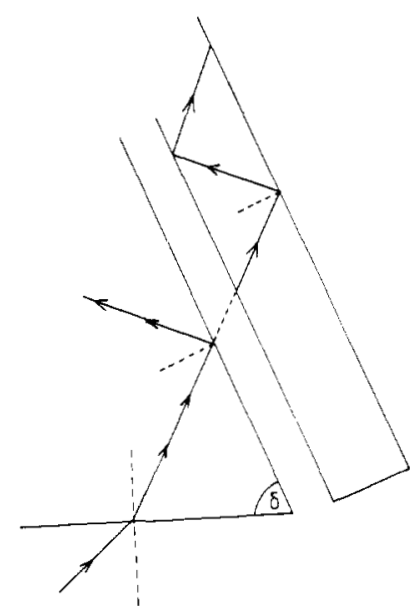

Figure 2. A prism coupler: a neutron suffering total reflection inside the Ti prism could enter into the Ti fibre by a tunnel effect across the air gap. Inside the fibre, there is confined neutron propagation.

critical angle upon $\Omega$ (see figure 1(b)). Should the waveguide be removed, so that confinement does not occur, a given neutron would have a transverse displacement $d \simeq z_{0} \tan i_{\mathrm{c}} \simeq 0.0157 \mathrm{~cm}$. Clearly, one would have a quite appreciable confining effect due to the waveguide when $R$ is smaller than $d$.

An alternative procedure for confining neutrons inside a solid waveguide which has a planar surface along the waveguide axis could consist of using a 'prism coupler' (see figure 2), as in integrated optics (see §II.D in Tien (1977)). Thus, one could imagine that the base of a Ti prism is strongly pressed against the planar surface of a Ti waveguide, leaving a small air (or vacuum) gap of width $10^{-5} \mathrm{~cm}$ between them. A neutron which has entered the prism is assumed to reflect totally at the base of the prism, so that only the evanescent field in the air gap could couple the neutron wave-function in the prism into the waveguide. This could be possible, in principle, since the neutron wave-function may be non-vanishing outside the base of the prism along a distance of the order of the air gap width (see § 3.2). Then, the neutron which would have entered into the waveguide by the tunnelling effect across the air (or vacuum) gap would propagate confined inside the waveguide.

\subsection{Cladded neutron fibres: a possibility for increasing the acceptance angle}

A possibility of increasing somewhat the confining effect consists in using 'cladded neutron fibres' (in the terminology of fibre optics). Here, one considers a cylindrical

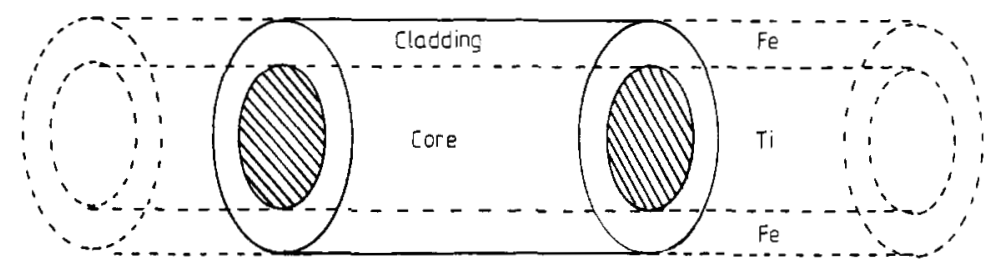

Figure 3. A possible cladded fibre: the inner Ti cylinder (core) is surrounded by an outer coaxial Fe cladding. 
solid waveguide (core) of an element from those listed in table 1, inside an outer coaxial cylinder (cladding), as shown in figure 3 for the case where the core and cladding are of $\mathrm{Ti}$ and $\mathrm{Fe}$, respectively. The element in the cladding should satisfy the following requirements: (i) large positive scattering amplitude, $b$, (ii) large number of nuclei per unit volume, $\rho$, (iii) small nuclear absorption. We note that iron fulfills all those requirements (see Bacon 1962, pp 31, 61). In this way, the net attraction felt by a neutron towards the inner core is somewhat enhanced. To assess this, let us consider the same situation as in figure $1(b)$, with the only difference being that the shaded regions correspond to the cladding material. Then equation $(2.2 .1), \delta=\pi / 2$, is replaced by

$$
\sin i_{c}=\frac{1}{n_{\mathrm{cl}}}\left[n_{\mathrm{co}}^{2}-n_{\mathrm{cl}}^{2}\right]^{1 / 2}
$$

where $n_{\mathrm{co}}, n_{\mathrm{cl}}$ are the refractive index for neutrons in the core and cladding respectively, as given by equation (2.1.3). For the case of a Ti core with a Fe cladding, at $E=0.02 \mathrm{eV}$, and using for Fe (Shull and Wollan 1951) $b=0.96 \times 10^{-12} \mathrm{~cm}$ and $\rho=8.48 \times 10^{22}$ nuclei $\mathrm{cm}^{-3}$, one finds $i_{\mathrm{c}} \simeq 3.61 \times 10^{-3} \mathrm{rad} \simeq 12.4^{\prime}$. That is, the acceptance angle increases by a factor 2.3. In the following text (except for discussions in $\S 3.2$ and $\S 4$ ), we shall always refer to fibres or solid waveguides in vacuum (without cladding), for simplicity.

\subsection{Reflectivity and evanescent wave for total internal reflection by a planar vacuum- fibre interface}

Let us consider again figure $1(a)$ with $\delta=\pi / 2$ (or the part of figure $1(b)$ where one internal reflection has taken place), and let us assume that the interface separating the unclad solid waveguide from the vacuum is reasonably flat. Then one can use the conventional theory summarised in Goldberger and Seitz (1947) for the reflectivity $\Re$ and for the depth $D_{\mathrm{ta}}$ for which the evanescent transmitted amplitude outside the waveguide (corresponding to total internal reflection inside it) falls to $e^{-1}$ times its value at the interface. By replacing $n$ by $n^{-1}$ in the corresponding equations given by Goldberger and Seitz (1947), we get:

$$
\begin{aligned}
& \Re=\left(\frac{\left(n^{-2}-\sin ^{2} i^{\prime}\right)^{1 / 2}-\cos i^{\prime}}{\left(n^{-2}-\sin ^{2} i^{\prime}\right)^{1 / 2}+\cos i^{\prime}}\right)^{2} \\
& D_{\mathrm{ta}}=\frac{\hbar}{(2 m E)^{1 / 2}\left(\sin ^{2} i^{\prime}-n^{-2}\right)^{1 / 2}} .
\end{aligned}
$$

Since $n>1$ for any element listed in table 1 and $i^{\prime}>i_{c}^{\prime}$ when total internal reflection occurs, using equation (2.2.1) we find $\Re=1$. On the other hand, by estimating the right-hand side of equation (2.4.2) for angles suitably larger than $i_{\mathrm{c}}^{\prime}$ (see Goldberger and Seitz 1947), one sees that $D_{\text {ta }}$ is of order $10^{2}$ to $10^{3} \AA$. The analogue of the actual $D_{\text {ta }}$ for the case of fibres with circular cross-section will be the quantity $\kappa^{-1}$, to be studied in $\$ 3.2$, the order of magnitude of which will be similar.

\subsection{Possible applications to radiotherapy}

At this point it may be noted that cancer treatments based upon slow-neutron capture by drugs containing ${ }^{10} \mathrm{~B}$ concentrated in malignant tumours suffer from the disadvantage that neutron doses can also reach the normal tissues of a patient (see Fowler 1981, $\S 4.2$ ). Then, a suitably cladded neutron fibre, having a suitably small cross-section 


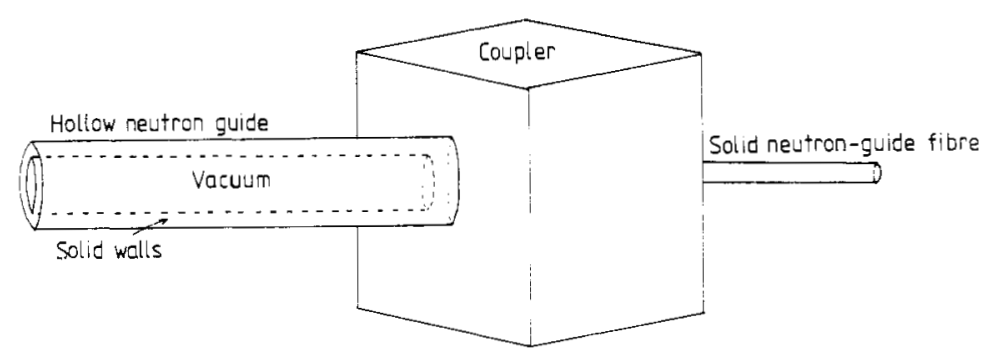

Figure 4. A standard hollow neutron guide connected, by a suitable coupling mechanism, to a non-hollow neutron-guide fibre of smaller transverse cross-section.

and a reasonable length (say, varying about $10 \mathrm{~cm}$, so that the neutron flux does not attenuate excessively due to nuclear absorption) and whose end is at a malignant tumour containing ${ }^{10} \mathrm{~B}$, could perhaps avoid the undesirable delivery of neutrons to tumour-free tissues, due to the confinement effect inside the solid waveguide. Moreover, the decrease of the flux of neutrons along the fibre could perhaps be not larger than that for a collimated neutron beam, such as those used in current radiotherapy (where there always occurs a loss of a certain percentage of the total neutron flux).

An arrangement which could perhaps have potential applications in slow-neutron radiotherapy is shown in figure 4 . A standard hollow neutron guide, having transverse cross-section of order several $\mathrm{cm}^{2}$ and guiding the neutrons from the reactor, is connected (by means of a suitable coupler-see $\$ 2.2$ ) to a non-hollow neutron fibre of smaller cross-section, which concentrates the neutron flux directly towards its end, which is located at a malignant tumour. For further comparative comments on hollow guides and non-hollow fibres, see $\$ 4$.

It may also be useful to consider curved neutron fibres for medical applications: for instance, in order to reach specific regions in a body which may not be easily accessible by means of a straight solid waveguide. Some standard concepts available for curved hollow neutron guides (length of direct view, characteristic angle, garland reflections, characteristic wavelength . . . see, for instance, Jacrot (1970)) can be directly carried over to curved neutron fibres.

Of course, neutron absorption would give rise to a gamma radiation background, and an important practical problem would consist in devising procedures in order to reduce it considerably. We shall not pursue this further, but we shall refer to $\$ 3.2 .1$ in Jacrot (1970), where methods for reducing the gamma background in hollow neutron guides are discussed. Clearly, in order to reduce gamma radiation, the fibre cross-section should be suitably small.

\section{Infinite homogeneous absorptionless fibre for neutron propagation}

\subsection{Propagation modes and quasiclassical estimate of their number}

We consider a given cylindrical homogeneous solid waveguide, formed of any material from those in table 1 (or of a suitable alloy containing at least one of them), so that the corresponding overall $b$ is real and negative (nuclear absorption being neglected). We shall always take the waveguide axis along the $z$ axis and denote its constant cross-section in the $x-y$ plane by $\Omega$, so that $x=(x, y, z)=(\bar{x}, z)$. In some general developments, the shape of $\Omega$ will be arbitrary, but in order to carry out numerical estimates, we shall take 
it later as circular for analytical convenience: such a choice will not alter the orders of magnitude substantially. Here, we shall assume that the waveguide extends from $z=$ $-\infty$ up to $z=+\infty$.

Under these conditions, a propagation mode for the neutron in the fibre is a solution of equation (2.1.1) such that

$$
\psi(\boldsymbol{x})=\exp (\mathrm{i} \beta z) \varphi_{\alpha}(\overline{\boldsymbol{x}})
$$

$\beta$ being the real propagation constant and $\alpha$ being a set of additional quantum numbers, and $\varphi_{a}(\overline{\boldsymbol{x}})$ is finite for any $\overline{\boldsymbol{x}}$, vanishes suitably as $|\overline{\boldsymbol{x}}| \rightarrow \infty$ and fulfills

$$
\begin{aligned}
& {\left[-\frac{\hbar^{2}}{2 m} \Delta_{\dot{x}}+V(\bar{x})\right] \varphi_{\alpha}(\bar{x})=-\frac{\hbar^{2}}{2 m} \kappa^{2} \varphi_{\alpha}(x) \quad \Delta_{\dot{x}}=\frac{\partial^{2}}{\partial x^{2}}+\frac{\partial^{2}}{\partial y^{2}}} \\
& E=\frac{\hbar^{2}}{2 m}\left(\beta^{2}-\kappa^{2}\right)
\end{aligned}
$$

where $K$ is real and positive and $V(\bar{x})$ is given by the right-hand side of equation (2.1.2) for $\bar{x}$ inside $\Omega$, while $V(\bar{x})=0$ for $\bar{x}$ outside it.

If $\Omega$ is not very small, one may expect a priori, that the total number $N$ of propagation modes (that is, of bound states in the two-dimensional potential $V(\bar{x})$ ) to be large. Then, the use of the quasiclassical approximation in order to provide an asymptotic estimate for $N$ is justified (see Martin 1972).

By including a factor two in order to count neutron propagation modes with both spin projections, the quasiclassical approximation yields:

$$
N \simeq \frac{2}{4 \pi} \int \mathrm{d}^{2} \bar{x}\left(-\frac{2 m}{\hbar^{2}} V(\bar{x})\right)=2 \rho(-b) A(\Omega)
$$

$A(\Omega)$ being the area of $\Omega$. Then, for typical values $\rho \simeq 10^{22}$ nuclei $\mathrm{cm}^{-3},-b \simeq$ $10^{-12} \mathrm{~cm}$ and $A(\Omega) \simeq 10^{-2} \mathrm{~cm}^{2}$, one has $N \simeq 10^{8}$ propagation modes. For the same values of $\rho,-b$ and a cross-section typical of a whisker $\left(A(\Omega) \approx 10^{-10} \mathrm{~cm}^{2}\right), N$ can be expected to lie between 1 and 10. In this case equation (3.1.4) cannot be applied with confidence.

Equation (3.1.4) gives that the number of propagation modes per unit area in the transverse cross-section of the fibre is a constant $(2 \rho(-b))$, whose order of magnitude is $10^{10} \mathrm{~cm}^{-2}$. On the other hand, in a typical thermal neutron beam, the flux $\Phi$ is about $10^{15}$ neutrons $\mathrm{cm}^{-2} \mathrm{~s}^{-1}$, and it may even be somewhat larger. Then, the number of neutrons which could enter into a solid waveguide in a given propagation mode (that is, with a given set of quantum numbers) may be about $10^{5} \mathrm{~s}^{-1}$ and perhaps more. Since the average thermal neutron velocity is $(2 E / m)^{1 / 2} \simeq 2 \times 10^{5} \mathrm{~cm} \mathrm{~s}^{-1}$ the (average) smallest distance between two neighbouring neutrons propagating along the fibre in the same propagation mode is of order $\left[(2 E / m)^{1 \cdot 2} / \Phi\right]^{1 / 3}$. For $\Phi$ about $10^{15}$ to $10^{18}$ neutrons $\mathrm{cm}^{-2} \mathrm{~s}^{-1}$, the latter distance varies between 60 and $600 \AA$, which is still considerably larger than the typical de Broglie wavelength for thermal neutrons $(1 \AA)$. Consequently, the wave packets associated to neutrons in a fibre in the same propagation mode have negligible overlap and can be treated as independent, that is, Fermi-Dirac statistics effects for them can be neglected. Notice, moreover, that nuclear absorption effects in the fibre (to be treated in $\$ 4$ ) will cause $\Phi$ to decrease as $z$ increases, so that Fermi-Dirac statistics effects will become increasingly negligible. 


\subsection{Fibre with circular cross-section: analysis of propagation modes}

For an infinite fibre with circular cross-section and radius $R$, equation (3.1.2) yields the following propagation modes in polar coordinates $(|\overline{\boldsymbol{x}}|, \phi)$;

$$
\begin{aligned}
& \varphi_{\alpha}(x)=\exp (\mathrm{i} M \phi) \begin{cases}c_{<} J_{|M|}\left(\boldsymbol{\kappa}^{\prime} \mid \overline{\boldsymbol{x}}\right) & |\overline{\boldsymbol{x}}|<R \\
c_{>} H_{(M \mid}^{(1)}(\mathrm{i} \kappa|\boldsymbol{x}|) & |\overline{\boldsymbol{x}}|>R\end{cases} \\
& \boldsymbol{\kappa}^{2}+\boldsymbol{\kappa}^{\prime 2}=-4 \pi b \rho
\end{aligned}
$$

where $J_{|\mathrm{M}|}$ and $H_{M}^{(1)}$ are the standard Bessel and outgoing Hankel functions of order $|M|, M=0, \pm 1, \pm 2, \ldots, c_{>}, c_{<}$are constants, and $\kappa^{\prime}$ is real and positive. For simplicity, we have omitted the spin wave functions $\left(\begin{array}{l}1 \\ 0\end{array}\right)$ or $\left(\begin{array}{l}0 \\ 1\end{array}\right)$ for the neutron in the right-hand side of equations $(3.2 .1-2)$ : throughout this paper, we shall also omit them. By imposing the continuity of $\varphi_{\alpha}(\overline{\boldsymbol{x}})$ and of its radial derivative at $|\overline{\boldsymbol{x}}|=R$, one gets the following dispersion relation for the propagation modes:

$$
\left[J_{|M|}\left(\kappa^{\prime} R\right)\right]^{-1}\left(\frac{\mathrm{d} J_{\mid M\left(K^{\prime}|\bar{x}|\right)}}{\mathrm{d}|\overline{\boldsymbol{x}}|}\right)_{\bar{x} \mid=R}=\left[H_{M}^{(1)}(\mathrm{i} \kappa R)\right]^{-1}\left(\frac{\mathrm{d} H_{\mid M}^{(1)}(\mathrm{i} \kappa|\overline{\boldsymbol{x}}|)}{\mathrm{d}|\overline{\boldsymbol{x}}|}\right)_{\mid \bar{x}=R}
$$

which, combined with equation (3.2.3), determines both $\kappa$ and $\varkappa^{\prime}$.

Notice that for typical values of $\rho,-b$ corresponding to the elements in table 1 , one has $(-4 \pi b \rho)^{1 / 2}$ is of order $10^{-2}$ or $10^{-3} \AA^{-1}$, so that $(-4 \pi \rho b)^{-1 / 2}=10^{2}$ to $10^{3} \AA$. Correspondingly, we find that $2 \pi \hbar^{2} \rho(-b) / m \simeq 10^{-8} \mathrm{eV}$. In spite of the smallness of such an energy, we notice that energy changes of order $10^{-8} \mathrm{eV}$ in neutron diffraction through perfect Si crystals in magnetic fields have been measured experimentally (see, for instance, Zeilinger and Shull (1979)).

We have analysed equations (3.2.3-4) for typical Ti values $\left((-4 \pi \rho b)^{1 / 2} \simeq 4 \times\right.$ $\left.10^{-3} \AA^{-1}\right), E \simeq 0.02 \mathrm{eV}$ (typical thermal neutron energy), $\beta \simeq 3 \AA^{-1} \gg(-4 \pi \rho b)^{1 / 2}$ and variable $R$. Thus, for $R \cong 10^{-5} \mathrm{~cm}$ (a typical whisker dimension) so that the number of propagation modes may lie between 1 and 10 , one finds that any allowed value for $|M|$ lies between 0 and $10, \kappa^{\prime}$ is quite close to (although smaller than) $(-4 \pi \rho b)^{1 / 2}$ and $K$ is very small ( $\geq 10^{-8} \AA^{-1}$ ). On the other hand, for $R \simeq 0.1 \mathrm{~cm}$ so that the number of propagation modes is very large $\left(\simeq 10^{8}\right)$, one finds that: (i) the two-dimensional ground state for equation (3.2.1) (that is, the propagation mode without nodes) corresponds to $M=0$, a value for $K$ quite close to (but smaller than) $(-4 \pi \rho b)^{1 / 2}$ and a very small $\kappa^{\prime}\left(\simeq 10^{-7} \AA^{-1}\right)$; (ii) the remaining propagation modes (excited eigenfunctions for equation (3.1.2)) correspond to increasing (decreasing) values of $\kappa^{\prime}(\kappa)$ and increasing $|M|$. An important property is that in all cases (that is, for either $K^{\prime} \ll K \leqslant(-4 \pi \rho b)^{1 / 2}$ or $K \ll K^{\prime} \leqslant(-4 \pi \rho b)^{1 / 2}$ or any intermediate case) $J_{|M|}\left(\kappa^{\prime}|\overrightarrow{\boldsymbol{x}}|\right)$ is practically a constant as $|\overline{\boldsymbol{x}}|$ varies in any interval whose total length is about $1 \AA$ : this follows from the general features of Bessel functions (for instance, from a rough estimate of the locations of their zeroes) and the fact that $\kappa^{\prime} \leqslant(-4 \pi \rho b)^{1 / 2} \ll 1 \AA^{-1}$. Use of this property will be made in $\S 5$.

By analogy with fibre optics (e.g. Marcuse 1972), it is easy to find the values of $R$ for which new propagation modes with $M \neq 0$ just become allowed ('cut-off conditions'). 
In fact, for given $M \neq 0$ and such a value of $R$, one has necessarily $\kappa=0$ for that propagation mode. By using

$$
\lim _{\kappa \rightarrow 0}\left[H_{M \mid}^{(1)}(\mathrm{i} \kappa R)\right]^{-1}\left(\frac{\mathrm{d} H_{M !}^{(1)}(\mathrm{i} \kappa|\bar{x}|)}{\mathrm{d}|\overline{\boldsymbol{x}}|}\right)_{\dot{\boldsymbol{x}} \mid=R}=-\frac{|M|}{R}
$$

and the recurrence relations 9.1.27 in Abramowitz and Stegun (1965) and equation (3.2.3), then equation (3.2.4) becomes the cut-off condition

$$
J_{M !-1}\left[(-4 \pi \rho b)^{1 / 2} R\right]=0 .
$$

For instance, for large enough $|M|$, one finds easily, using the asymptotic expansion 9.5.14 in Abramowitz and Stegun (1965), that a new propagation mode with such a value for $M$ just occurs if $R \simeq|M|(-4 \pi \rho b)^{-1 / 2}$, etc.

If the above fibre of circular cross-section is not surrounded by vacuum but by an infinite medium made of an element with positive nuclear scattering amplitude $\left(b_{\mathrm{cl}}>0\right)$ and $\rho_{\mathrm{cl}}$ nuclei per unit volume, one has

$$
V(\overline{\boldsymbol{x}})=\frac{2 \pi \hbar^{2}}{m} \rho_{\mathrm{sl}} b_{\mathrm{sl}} \text { for } \quad|\overline{\boldsymbol{x}}|>R
$$

Then equations (3.2.1) and (3.2.3) still hold, but one should replace $H_{M}^{(1)}(\mathrm{i} K|\bar{x}|), H_{\mid M}^{(1)}(\mathrm{i} K R)$ in equations (3.2.2) and (3.2.4) by $H_{\mid M}^{(1)}\left(\mathrm{i} \kappa_{\mathrm{cl}}|\bar{x}|\right), H_{|M|}^{(1)}\left(\mathrm{i} \kappa_{\mathrm{cl}} R\right)$, where $-\kappa_{\mathrm{ci}}^{2}+\kappa^{2}=-4 \pi \rho_{\mathrm{cl}} b_{\mathrm{cl}}$. Then, the generalisation of the study previously made for the unclad fibre to the one with infinite cladding is straightforward.

\subsection{Magnetised fibre: a short discussion}

We outline the generalisation to neutron propagation inside a magnetised homogeneous medium, still omitting other effects (lattice periodicity and vibrations, ...) for simplicity. Then, by following Halpern et al 1941 (see also Lax 1951, Hughes 1954, Bacon 1962), equations (2.1.2) should be replaced by

$$
V(\overline{\boldsymbol{x}})=\frac{2 \pi \hbar^{2} \rho}{m}\left(b \mathbf{I}+b_{\mathrm{mag}} \boldsymbol{u} \boldsymbol{\sigma}\right)
$$

where $b_{\text {mag }}$ is the magnetic scattering amplitude, $u$ is a unit vector in the direction of the saturated magnetic induction. $I$ is the unit $2 \times 2$ matrix and $\sigma$ is the standard vector formed by the Pauli matrices

Instead of equation (2.1.3), we now have:

$$
n^{2}=1-\frac{4 \pi \rho}{K^{2}}\left(b \pm b_{\mathrm{mag}}\right)
$$

where the sign $+(-)$ corresponds to neutron spin parallel (antiparallel) to $u$. An interesting example is ${ }^{59} \mathrm{Co}$ for which $b=+0.28 \times 10^{-12} \mathrm{~cm} ; b_{\text {mag }} \simeq 0.47 \times 10^{-12} \mathrm{~cm}$ (see Bacon 1962, p 171) so that $b-b_{\text {mag }}<0, b+b_{\text {mag }}>0$ and, hence, there would be propagation modes in a Co fibre when the neutron spin and the saturated magnetic induction are antiparallel, but none when they are parallel. In the former case, for a cobalt $\left({ }^{59} \mathrm{Co}\right)$ fibre whose cross-section is not small, the quasiclassical approximation allows us to estimate the total number of neutron propagation modes (equation (3.1.4)) as $\rho\left(-b+b_{\text {mag }}\right) A(\Omega)$. Let us consider a magnetised Co solid waveguide, such that $\boldsymbol{u}$ lies along the positive $z$ axis. Then, provided that $b$ in equation (2.1.2) is replaced by $b-b_{\text {mag }}$, the analysis in $\$ 3.2$ can be directly extended to the actual 
propagation modes, in which the neutron spin is antiparallel to $\boldsymbol{u}$. Magnetised mirrors of cobalt have been used to produce polarised neutrons (see Hughes 1954, p 128, and Hughes and Burgy 1951). One drawback of a magnetised Co fibre would be the comparatively large attenuation suffered by a neutron beam along the former, due to nuclear absorption: in fact, the linear absorption coefficient for Co is $1.87 \mathrm{~cm}^{-1}$ (see Bacon 1962, p 61, and compare with table 2 in this paper). In other words, a magnetised Co fibre where confined propagation of polarised neutrons would occur would seem possible, but its length could be expected to be about one order of magnitude smaller than that of a possible Ti fibre.

\subsection{Summary}

In the previous subsections of this section, we have analysed the possible propagation modes for a neutron along an idealised homogeneous fibre, i.e. the possible waves propagating along the waveguide axis which are confined in the directions transverse to the latter. The fibre was assumed to be made of any element from those listed in table 1 , and to have infinite length and circular cross-section. The analysis was made on the basis of the Schrodinger equation and is analogous in spirit to that for propagation modes of light in an optical fibre (Marcuse 1972). Nuclear absorption and lattice periodicity have so far been disregarded. The main results are: (i) the quasiclassical estimate of the number of propagation modes (equation (3.1.4)) and a qualitative understanding of how a typical neutron flux distributes among the latter; (ii) the explicit expressions for the neutron propagation modes (equations (3.2.1-4)); (iii) approximate numerical estimates of some relevant quantities $\left(\kappa, \kappa^{\prime}\right)$ and the observation that the wave-function associated with any propagation mode is practically a constant as $\overline{\boldsymbol{x}}$ varies in the transverse cross-section over distances of order $1 \AA$; (iv) in a $C o$ fibre magnetised along the waveguide axis (positive $z$ axis) any confined neutron travelling in a confined propagation mode is polarised (its spin points along the negative $z$ axis).

\section{Homogeneous fibre with nuclear absorption}

We now consider a cylindrical homogeneous fibre similar to that treated in $\S 3$, except for the following two differences: (i) its extension along the $z$ axis is restricted to $0<z<z_{0}$, where either $z_{0}=+\infty$ (semi-infinite fibre) or $z_{0}$ is finite and much larger than any length characterising the fibre cross-section $\Omega$; (ii) nuclear absorption is now allowed for, that is, $\operatorname{Re} b<0, \operatorname{Im} b \leqslant 0$ and, hence, equation (2.1.2) implies that $\operatorname{Im}$ $V \leqslant 0$ inside the fibre.

In the present situation, a generalised propagation mode in the fibre region $0<$ $z<z_{0}$ is a solution of equation (2.1.1) for this interval, fulfilling formally the same equations (3.1.1-3) but with the following new specific conditions: $E>0, \beta$ is complex with $\operatorname{Re} \beta>0$ and $\kappa$ is also complex, with $\operatorname{Re} K>0$. We stress that the actual $\varphi_{\alpha}(\bar{x})$ is finite for any $\bar{x}$ and vanishes quickly as $|\overline{\boldsymbol{x}}| \rightarrow \infty$.

For the actual complex $V$, a physically interesting problem is to evaluate the corresponding values of $\operatorname{Im} \beta$, since they determine how the generalised propagation modes for the neutron attenuate as $z$ increases in $0<z<z_{0}$. Note that by taking imaginary parts in the analogue of equation (3.1.3) for the actual case, one gets 


$$
\operatorname{Im} \beta=(2 \operatorname{Re} \beta)^{-1} \operatorname{Im}\left(\beta^{2}\right)=(2 \operatorname{Re} \beta)^{-1} \operatorname{Im}\left(\kappa^{2}\right)
$$

It will be useful to introduce the notations:

$$
\nabla_{\bar{x}}=\left(\frac{\partial}{\partial x}, \frac{\partial}{\partial y}\right) \quad\left(\varphi_{1}, \varphi_{2}\right)=\int \mathrm{d}^{2} \bar{x} \varphi_{1}(\overline{\boldsymbol{x}})^{*} \varphi_{2}(\overline{\boldsymbol{x}})
$$

for any $\varphi_{1}(\overline{\boldsymbol{x}}), \varphi_{2}(\overline{\boldsymbol{x}})$ which vanish appropriately as $|\overline{\boldsymbol{x}}| \rightarrow \infty$ (the integration extends over the whole $\bar{x}$ plane) and $\|\varphi\|=|(\varphi, \varphi)|^{1 / 2}$. Then, the analogue of equation (3.1.2) for the present situation yields readily:

$\left[\varphi_{\alpha},\left(-\frac{\hbar^{2}}{2 m} \Delta_{\dot{x}}+V\right) \varphi_{\alpha}\right]=\frac{\hbar^{2}}{2 m}\left\|\nabla_{\dot{x}} \varphi_{\alpha}\right\|^{2}+\left(\varphi_{\alpha}, V \varphi_{\alpha}\right)=-\frac{\hbar^{2}}{2 m} \kappa^{2}\left\|\varphi_{\alpha}\right\|^{2}$

Notice that a partial integration has been performed. By taking imaginary parts in equation (4.3), we get

$$
\begin{aligned}
\operatorname{Im}\left(\kappa^{2}\right)=- & \frac{2 m}{\hbar^{2}}\left(\left\|\varphi_{\alpha}\right\|^{2}\right)^{-1}\left(\varphi_{\alpha}, \operatorname{Im} V \varphi_{\alpha}\right) \\
& =-\frac{2 m}{\hbar^{2}}(\operatorname{Im} V)\left(\left\|\varphi_{\alpha}\right\|^{2}\right)^{-1} \int_{\Omega} \mathrm{d}^{2} \overline{\boldsymbol{x}}\left|\varphi_{\alpha}(\overline{\boldsymbol{x}})\right|^{2} .
\end{aligned}
$$

The last expression in equation (4.4) follows from the fact that Im $V$ is constant (vanishes) inside (outside) $\Omega$.

\subsection{Consequences}

(i) As $\operatorname{Im} V \leqslant 0$ inside the fibre, equation (4.4) implies $\operatorname{Im}\left(\kappa^{2}\right) \geqslant 0$.

(ii) Since $\Omega$ is a finite domain in the $\bar{x}$ plane, one has

$$
\int_{\Omega} \mathrm{d}^{2} \overline{\boldsymbol{x}}\left|\varphi_{\alpha}(\overline{\boldsymbol{x}})\right|^{2} \leqslant\left\|\varphi_{\alpha}\right\|^{2}
$$

and then equation (4.4) implies

$$
\operatorname{Im}\left(\kappa^{2}\right) \leqslant \frac{2 m}{\hbar^{2}}(-\operatorname{Im} V) .
$$

(iii) Equation (4.1) together with the fact that $\operatorname{Re} \beta>0$ and the above consequences (i) and (ii) give successively

$$
\operatorname{Im} \beta>0 \quad \operatorname{Im} \beta \leqslant \frac{m}{\hbar^{2} \operatorname{Re} \beta}(-\operatorname{Im} V) .
$$

(iv) The second inequality (4.5) and equation (2.1.2) yield

$$
\operatorname{Im} \beta \leqslant \frac{2 \pi}{\operatorname{Re} \beta} \rho(-\operatorname{Im} b)=\frac{1}{2} \rho \sigma_{\mathrm{a}} .
$$

(v) The optical theorem for the individual neutron-nucleus scattering at very low energy with total cross-section $\sigma_{\mathrm{tot}}\left(\sigma_{\mathrm{tot}} \geqslant(4 \pi / \operatorname{Re} \beta)(-\operatorname{Im} b)\right.$, see Sears $\left.(1982)\right)$, together with the inequality (4.6) implies:

$$
\operatorname{Im} \beta \leqslant \rho \frac{\sigma_{\mathrm{tot}}}{2} \equiv \frac{\mu}{2}
$$

$\mu$ being the linear absorption coefficient in the usual notation, which also includes the 
contribution from diffuse scattering (Sears 1982). Cross-sections from which $\mu$ can be derived are available for many isotopes in Maghabghab et al (1981). Equation (4.6) gives a bound for $\operatorname{Im} \beta$ in terms of $\frac{1}{2} \rho$ times the absorption cross-section $\sigma_{\mathrm{a}}$ which is more restrictive than (4.7). The values for the product $\rho \sigma_{\mathrm{a}}$ for some elements listed in table 1 are given by Bacon (1962 pp 61-2). They are reproduced in table 2, and denoted by $\mu$ as well, in order to agree with Bacon's notation. Notice that we have included the value of $\mu$ for $\mathrm{Ni}$ (averaged over isotopes) instead of that for ${ }^{62} \mathrm{Ni}$, which is presumably unknown (to the authors' knowledge). The quoted values of $\mu$ correspond to a neutron wavelength $2 \pi \hbar(2 m E)^{-1 / 2}=1.08 \AA$. The interpretation of $\mu$ as $\rho \sigma_{\text {tot }}$ (instead of as $\rho \sigma_{\mathrm{a}}$ ) as a bound for $2 \operatorname{Im} \beta$ implies a somewhat larger attenuation, but does not qualitatively our results.

Another point of view consists in (i) regarding $\operatorname{Im} V$ as a perturbation of $-\left(\hbar^{2} / 2 m\right) \Delta_{\dot{x}}+\operatorname{Re} V$ and, hence, of its eigenfunctions and real eigenvalues, to be denoted here as $\varphi_{\alpha}^{(0)}$ and $-\left(\hbar^{2} / 2 m\right)\left[\kappa^{(0)}\right]^{2}$ respectively (given through equations (3.2.1-4)); (ii) applying standard Rayleigh-Schrödinger or Brillouin-Wigner perturbation theory in order to obtain $\operatorname{Im}\left(\kappa^{2}\right)$ and, hence, using equation (4.1), $\operatorname{Im} \beta$. The justification for all these comes from the fact that $(\operatorname{Re} V)^{-1} \operatorname{Im} V=(\operatorname{Re} b)^{-1} \operatorname{Im} b$ is usually very small (see Sears 1982).

First-order Rayleigh-Schrödinger perturbation theory and equation (4.1) give readily (assuming $\left\|\varphi_{\alpha}^{(0)}\right\|=1$ ):

$$
\operatorname{Im} \beta \simeq \frac{2 \pi \rho}{\operatorname{Re} \beta}(-\operatorname{Im} b) \int_{\Omega} \mathrm{d}^{2} \overline{\boldsymbol{x}}\left|\varphi_{\alpha}^{(0)}(\overline{\boldsymbol{x}})\right|^{2}
$$

which is consistent with equation (4.4) and its consequences.

For those propagation modes which are very concentrated inside the fibre ( $K$ close to $(-4 \pi \rho b)^{1 / 2}, \boldsymbol{\kappa}^{\prime}$ very small), one has $\int_{\Omega} \mathrm{d}^{2} \tilde{\boldsymbol{x}}\left|\varphi_{\alpha}^{(0)}(\overline{\boldsymbol{x}})\right|=1$, so that the inequality (4.6) is almost saturated and $\operatorname{Im} \beta$ and, hence, the attenuation due to nuclear absorption are the largest. This result is consistent with other calculations of the linear absorption coefficient for a neutron beam across a planar slab (see $\$ 6.4$ in Sears (1982) and references therein). On the other hand, for a propagation mode which is not very deeply concentrated inside the fibre, $|M|$ may be relatively large, $\kappa^{\prime}$ may be relatively close to $(-4 \pi \rho b)^{1 / 2}$ and $K$ rather small. Then, $\int_{\Omega} \mathrm{d}^{2} \overline{\boldsymbol{x}}\left|\varphi_{\alpha}^{(0)}(\overline{\boldsymbol{x}})\right|^{2}$ may be less than urity and so $\operatorname{Im} \beta$ and the attenuation coming from nuclear absorption may be somewhat reduced.

Let us assume that free thermal neutrons with wave-vector $k=(\bar{k} \beta)$ and flux $\Phi_{\mathrm{T}}$ produced at a nuclear reactor in a Maxwellian distribution, approach the start of the fibre at $z=0$. We shall extend directly equations (1) and $(4 a-b)$ in Schaerpf and Eichler (1973) for the case of a straight (non-curved) fibre. We find the following qualitative result for the spectral density of neutrons per unit area at $z=0(\lambda=$ $2 \pi / \beta=$ de Broglie wavelength for neutrons):

$$
\frac{2 \pi \Phi_{\mathrm{T}}}{K_{\mathrm{T}}^{4}} \frac{\exp \left[-\left(\lambda_{\mathrm{T}} / \lambda\right)^{2}\right]}{\lambda^{3}} \int \mathrm{d}^{2} \bar{k} \simeq \frac{2 \pi \Phi_{\mathrm{T}}}{K_{\mathrm{T}}^{4}} \frac{\exp \left[-\left(\lambda_{\mathrm{T}} / \lambda\right)^{2}\right]}{\lambda^{3}} \cdot 4^{2} \pi \rho(-b)
$$

$K_{\mathrm{B}} T=\left(\hbar^{2} / 2 m\right) K_{\mathrm{T}}^{2}, K_{\mathrm{T}}=2 \pi / \lambda_{\mathrm{T}}, K_{\mathrm{B}}$ and $T$ being Boltzmann's constant and the absolute temperature, respectively. The integral $\int \mathrm{d}^{2} \overrightarrow{\boldsymbol{k}}$ in the left-hand side of equation (4.9) was extended only over those values of $k$ such that total internal reflection occurs inside the waveguide $(\$ 2.2)$, so that its value is given approximately for a fibre with rectangular cross-section, by $4 \beta^{2}\left(\tan i_{\mathrm{c}}\right)^{2} \simeq 4^{2} \pi \rho(-b)$. The results for other shapes of the transverse cross-section have the same order of magnitude. 
Table 2. Linear absorption coefficient, $\mu$, for the elements listed in table 1 .

\begin{tabular}{llllll}
\hline & \multicolumn{5}{c}{ Element } \\
\cline { 2 - 6 } & $\mathrm{Li}$ & $\mathrm{Ti}$ & $\mathrm{V}$ & $\mathrm{Mn}$ & $\mathrm{Ni}$ \\
\hline$\mu\left(\mathrm{cm}^{-1}\right)$ & 1.87 & 0.20 & 0.20 & 0.60 & 0.25 \\
\hline
\end{tabular}

For $z>0$, the flux associated with a pure state such as the generalised propagation mode $\psi$ (or a superposition thereof, such as that in equation (A2.3)) can be obtained by integrating the quantum-mechanical current along the $z$ axis, namely

$$
\left.\frac{\hbar}{m} \operatorname{Re}\left[\psi^{*}\left(-\mathrm{i} \frac{\partial}{\partial z}\right) \psi\right]\right]
$$

across an infinite surface orthogonal to the fibre axis. Such a flux, when the incoming neutrons approaching the fibre at $z=0$ are represented by the Boltzmann distribution (a statistical mixture), has to be averaged suitably over the latter. By approximating $2 \operatorname{Im} \beta \simeq \mu$, a rough estimate yields the physically expected result for the spectral density at $z \geqslant 0$ :

$$
\frac{2 \pi \Phi_{\mathrm{T}}}{K_{\mathrm{T}}} \frac{\exp \left[-\left(\lambda_{\mathrm{T}} / \lambda\right)^{2}\right]}{\lambda^{3}} \times 4 \beta^{2}\left(\tan i_{\mathrm{c}}\right)^{2} \exp (-\mu z) .
$$

The main factors in equation (4.10) which give rise to a reduction in the neutron flux are $\left(\tan i_{\mathrm{c}}\right)^{2}$ and $\exp (-\mu z)$, whose physical origins are the confinement effect and nuclear absorption, respectively. The order of magnitude of $\left(\tan i_{\mathrm{c}}\right)^{2}$ is about $10^{-5}$ to $10^{-6}$, whereas that of $\exp (-\mu z)$ for $z=20-30 \mathrm{~cm}$ is $10^{-2}$ to $10^{-3}$ (for $\mu \simeq 0.2 \mathrm{~cm}^{-1}$ ).

To summarise the main results so far presented in this section. (i) We have outlined a theoretical determination of $\operatorname{Im} \beta$ and have obtained specific bounds (equations $(4.6-7)$ ) and perturbation theory results (equation (4.8)): the latter shows that the attenuation due to nuclear absorption is reduced for those propagation modes which are not too concentrated inside the fibre (small $k$ ). As a first approximation, our results are consistent with previous studies by other authors. (ii) We have outlined an approximate formula (equation (4.10)) for the spectral density in the fibre when the incoming neutrons approaching it are represented by a Boltzmann distribution.

Table 3 summarises a qualitative comparison between the standard hollow guides

Table 3. Comparison of hollow and non-hollow neutron guides

\begin{tabular}{ll}
\hline Standard hollow neutron guides & Neutron fibres \\
\hline $\begin{array}{l}\text { Neutrons guided over long distances } \\
\text { (hundreds of meters) }\end{array}$ & $\begin{array}{l}\text { Confined neutron propagation over } \\
\text { short distances (about } 10 \mathrm{~cm} \text { ) to } \\
\text { avoid drastic reductions of flux due } \\
\text { to nuclear absorption }\end{array}$ \\
$\begin{array}{l}\text { A hollow tube: walls made of } \\
\text { certain materials and vacuum inside }\end{array}$ & $\begin{array}{l}\text { A solid (non-hollow) cylindrical } \\
\text { sample of an element listed in table } \\
1(\mathrm{e} . \mathrm{g} . \text { Ti): no vacuum region inside } \\
\text { the fibre }\end{array}$ \\
$\begin{array}{l}\text { Relatively large transverse cross- } \\
\text { sections (of order several } \mathrm{cm}^{2} \text { ) }\end{array}$ & Small transverse cross-section \\
\hline
\end{tabular}


and the neutron fibres or solid waveguides proposed in this paper. The net conclusion is that the latter are not intended to replace the former for neutron confined propagation over long distances. Rather, a non-hollow neutron fibre of very small crosssection and with a length of about $10 \mathrm{~cm}$ connected or coupled to a hollow guide (see figure 4), may be useful in order to concentrate neutrons in a very small region, whose dimensions are smaller than the transverse cross-section of a typical hollow guide (of order $\mathrm{cm}^{2}$ ).

The construction of a hollow neutron guide of very small cross-section and small losses would also constitute, in itself, an interesting possibility (in particular, in connection with radiotherapy). On the other hand, in order to reduce losses in the flux of neutrons (and, hence, to achieve solid waveguides of somewhat greater length), and as a possible alternative to the cladded fibres treated in $\$ 2.3$, one could replace the core and clad in figure 3 by other elements having both positive nuclear scattering amplitudes $b_{\mathrm{co}}$ (core) $b_{\mathrm{cl}}$ (cladding), with the following conditions: (i) $0<b_{\mathrm{co}}<b_{\mathrm{cl}}, n_{\mathrm{co}}>n_{\mathrm{cl}}$, so that the acceptance angle should be not too small; (ii) the nuclear absorption (and the total cross-section for the scattering) of neutrons by the nuclei of the core material should be small; (iii) the penetration depth for neutrons in the cladding $\left(10^{2}-10^{3} \AA\right)$ should be much smaller than its 'width' (namely, the difference between the external radius of the cladding and the radius of the core), so that the probability for a neutron to go from the core to vacuum by tunnel effect across the clad should be negligible. For instance, the core could be made up of $\mathrm{Si}$ (the waveguide cross-section being non-circular). Another possibility would be to use the same materials for core and cladding (aluminium and nickel, respectively) that have already been used in the neutron microguides (alternate planar layers of $\mathrm{Al}$ and Ni) described by Marx (1971).

\section{Infinite periodic absorptionless neutron fibres}

In the previous sections, we have analysed neutron confinement and propagation modes inside fibres made of elements from those listed in table 1, disregarding completely lattice periodicity effects.

The question then arises, whether such a picture is modified when lattice periodicity and, eventually, vibration effects in neutron fibres are taken into account. It seems important to provide some answers to such a question. The analysis of vibration effects in crystal lattices of elements listed in table 1 given in appendix 1 shows that they can be disregarded for ordinary temperatures. This property allows one to concentrate solely on the effects of lattice periodicity upon the neutron propagation modes, to which $\S 5$ is devoted. After some simplifications, the main results show (§5.3) (i) wide allowed and small forbidden bands for the neutron energy along the periodic fibre; (ii) well inside an allowed band, the neutron propagation modes coincide, as a first approximation, with those for the homogeneous fibre.

\subsection{Potential and propagation modes: general aspects}

We shall treat an infinite cylindrical fibre similar to the one described in $\$ 3.1$, with the following specific differences:

(i) It is made from a perfectly crystallised material with basic lattice vectors $a_{1}$, $a_{2}$ and $a_{3}$, so that $a_{3}$ is parallel to the fibre $z$ axis. We shall suppose that $a_{3}$ is 
orthogonal to both $a_{1}$ and $a_{2}$ : this restriction is not essential, although convenient, and is valid for both $\mathrm{Ti}$ and $\mathrm{Mn}$ waveguides. Then, one has $\boldsymbol{a}_{i}=\left(\bar{a}_{i}, 0\right), i=1,2$.

(ii) $V(\boldsymbol{x})=0$ for $\boldsymbol{x}$ outside the fibre, while for $\boldsymbol{x}=(\overline{\boldsymbol{x}}, z)$ inside it the potential confining the neutron is

$V(\boldsymbol{x})=\frac{2 \pi \hbar^{2} b}{m} \sum_{l_{3}=-\infty}^{\infty} \sum_{l_{1}, l_{2}} \sum_{d} \delta^{(2)}\left[\overline{\boldsymbol{x}}-\sum_{i=1}^{2}\left(l_{i}+d_{i}\right) \overline{\boldsymbol{a}}_{i}\right] \delta\left[z-\left(l_{3}+d_{3}\right)\left|\boldsymbol{a}_{3}\right|\right]$

where $b$ is real $(b<0)$, the integers $l_{1}, l_{2}$ vary in a large, but finite region $\left(\left|l_{i}\right|<+\right.$ $\infty, i=1,2)$ and

$$
\boldsymbol{d}=\sum_{i=1}^{3} d_{j} \boldsymbol{a}_{j}
$$

where $d_{j}, j=1,2,3$ are certain numbers with $0 \leqslant\left|d_{j}\right|<1$ (see $\S 2.1$ in Marshall and Lovesey (1971) or appendix 1). Notice that equation (5.1.1) is the natural restriction of equation (A1.1) (valid for a crystal occupying the whole three-dimensional space) to the actual fibre having infinite length along the $z$ axis but finite cross-section $\Omega$.

The developments in Appendix 1 show that $V(x)$, as given by equation (5.1.1), may be regarded as an acceptable approximation for the potential seen by a neutron at ordinary temperatures $(=300 \mathrm{~K})$.

In the infinite periodic fibre, a propagation mode is naturally defined to be a solution of equation (2.1.1) for the actual $V(x)$ such that

(i)

$$
\psi(\boldsymbol{x})=\exp (\mathrm{i} \beta z) U_{\alpha}(\overline{\boldsymbol{x}}, z) \quad \beta \text { being real }
$$

(ii) $U_{\alpha}(\overline{\boldsymbol{x}}, z)$ is finite for any $\overline{\boldsymbol{x}}, z$, vanishes exponentially as $|\overline{\boldsymbol{x}}| \rightarrow \infty$ for given $z$, is periodic in $z$ (with period $\left|\boldsymbol{a}_{3}\right|$ ) for given $\overline{\boldsymbol{x}}$ and fulfills:

$$
\left[-\frac{\hbar^{2}}{2 m} \Delta_{\bar{x}}-\frac{\hbar^{2}}{2 m}\left(\frac{\partial}{\partial z}+\mathrm{i} \beta\right)^{2}+V(x)\right] U_{\alpha}(x, z)=E U_{\alpha}(\bar{x}, z) .
$$

\subsection{Further simplification of the potential seen by a neutron}

We now justify the assumption that the functions $U_{\alpha}(\bar{x}, z)$ characterising the neutron propagation modes are practically constant as $\overline{\boldsymbol{x}}$ varies over distances of order $\overline{\boldsymbol{a}}_{1}, \overline{\boldsymbol{a}}_{2}$ (of order $1 \AA$ ) for fixed $z$, so that the potential $V(x)$ in equation (5.1.1) can be replaced by a simpler one (see equation (5.2.9)):

We consider a fibre with circular cross-section and, as a first approximation, for $\overline{\boldsymbol{x}}$ inside the fibre we replace $V(\boldsymbol{x})$ (equation (5.1.1)) by

$$
V_{0}(x)=\frac{2 \pi \hbar^{2} b n(\{d\})}{m\left[\left(a_{1} \times a_{2}\right) a_{3}\right]}
$$

where $n(\{d\})$ is the total number of different $\boldsymbol{d}$ values. The right-hand side of equation (5.2.1) coincides with:

(i)

$$
\left[\left(a_{1} \times a_{2}\right) a_{3}\right]^{-1} \int_{\text {unit cell }} \mathrm{d}^{3} x V(x)
$$

the integral being performed over a unit cell well inside the fibre (far from its boundary) 
and $V(\boldsymbol{x})$ being given in equation (5.1.1); (ii) the right-hand side of equation (2.1.2), as $\rho=\left[\left(\boldsymbol{a}_{1} \times \boldsymbol{a}_{2}\right) \boldsymbol{a}_{3}\right]^{-1} \cdot n(\{d\})$; (iii) the $\boldsymbol{\tau}=0$ contribution in equation (A1.8), when $\Lambda=0$. Consequently, the propagation modes and the functions $U_{\alpha}^{(0)}$ for $V_{0}(x)$ are obtained immediately by comparing equations (5.1.2), (3.1.1) and (3.2.1-2), while $E$ is given by equation (3.1.3).

It is natural to decompose $V(\boldsymbol{x})$, as given by equation (5.1.1) for $\boldsymbol{x}$ inside the fibre, as:

$$
V(\boldsymbol{x})=V_{0}(\boldsymbol{x})+V_{1}(\boldsymbol{x})
$$

so that $V_{1}(\boldsymbol{x})$ can be regarded as a perturbation of $V_{0}(\boldsymbol{x})$ and of its propagation modes, and to obtain those for $V(\boldsymbol{x})$, in which we are interested, by applying BrillouinWigner perturbation theory. For any $\boldsymbol{x}$ inside the fibre, one has:

$$
\begin{aligned}
& V_{1}(\boldsymbol{x})=V_{1.1}(z)+V_{1,2}(\boldsymbol{x}) \\
& V_{1.1}(z)=\left|\overline{\boldsymbol{a}}_{1} \times \overline{\boldsymbol{a}}_{2}\right|^{-1} \int_{\text {unit shell }} \mathrm{d}^{2} \overline{\boldsymbol{x}} V_{1}(\boldsymbol{x})
\end{aligned}
$$

the integral in equation (5.2.4) being performed over the unit shell determined by $a_{1}$, $a_{2}$ in the $\bar{x}$ plane (that is, the intersection of the basic unit cell and the $\bar{x}$ plane). By considering equation (A1.8) for the infinite three-dimensional lattice when $\Lambda=0$, it follows that, for the fibre, one has approximately:

$$
\begin{gathered}
V_{1,2}(\boldsymbol{x}) \simeq \frac{2 \pi \hbar^{2} b}{m\left[\left(\boldsymbol{a}_{1} \times \boldsymbol{a}_{2}\right) \boldsymbol{a}_{3}\right]} \sum_{s_{1}, s_{2}} \sum_{s_{3}} \sum_{d} \exp \left[\mathrm{i}\left(\sum_{j=1}^{3} s_{j} \boldsymbol{\tau}_{j}\right)(\boldsymbol{x}-\boldsymbol{d})\right] \\
\left(s_{1}, s_{2}\right) \neq(0,0)
\end{gathered}
$$

where $s_{j}$ are integers and $\tau_{j}$ are the reciprocal lattice vectors. We introduce the scalar product:

$$
\left(U_{1}, U_{2}\right)_{1} \equiv \int_{-\mid a_{3} / 2}^{a_{3} / 2} \mathrm{~d} z \int \mathrm{d}^{2} \bar{x} U_{1}(\bar{x}, z)^{*} U_{2}(\bar{x}, z)
$$

where $U_{i}(\bar{x}, z), i=1,2$, are finite, for any $\bar{x}, z$ and, for given $\bar{x}$, periodic in $z$ with period $\left|a_{3}\right|$. Notice that $\int \mathrm{d}^{2} \overline{\boldsymbol{x}}$ is extended over the whole $\overline{\boldsymbol{x}}$ plane, so that $U_{i}$ has to vanish suitably as $|\overline{\boldsymbol{x}}| \rightarrow \infty$. Let $\mathbb{P}_{\alpha}^{(0)}, 1$ be the projector upon $U_{\alpha}^{(0)}=U_{\alpha}^{(0)}(\overline{\boldsymbol{x}}, z)$ and the unit operator, and let $\left[E-H_{0}\right]^{-1}$ be the resolvent of the operator

$$
H_{0} \equiv-\frac{\hbar^{2}}{2 m} \Delta_{\vec{x}}-\frac{\hbar^{2}}{2 m}\left(\frac{\partial}{\partial z}+i \beta\right)^{2}+V_{0}(\boldsymbol{x})
$$

Then, Brillouin-Wigner perturbation theory yields the following exact equations for $U_{\alpha}(\bar{x}, z), E$ (which satisfy equation (5.1.3)) in terms of $U_{\alpha}^{(0)}(\bar{x}, z), E^{(0)}$ (which fulfil equations $(5.1 .2-3),(3.1 .1),(3.2 .1-2)$, for $\left.V=V_{0}\right)$ :

$$
\begin{aligned}
& U_{\alpha}=U_{\alpha}^{(0)}+\left[\mathbb{1}-\mathbb{P}_{\alpha}^{(0)}\right]\left[E-H_{0}\right]^{-1} V_{1} U \\
& E=E^{(0)}+\left(U_{\alpha}^{(0)}, V_{1} U_{\alpha}\right)_{1} .
\end{aligned}
$$

By considering the first-order correction or, more generally, any relevant matrix element contributing to the successive iterations of equations (5.2.7-8), one is led to study $\left(U_{\alpha^{\prime}}^{(0)},\left(V_{1,1}+V_{1.2}\right) U_{\alpha^{\prime}}^{(0)}\right)_{1}$. Let us recall, (i) equations (5.1.2), (3.1.1), (3.2.1); (ii) the fact (discussed in $\$ 3.2$ ) that $J_{|M|}\left(\kappa^{\prime}|\bar{x}|\right)$ is practically constant as $\bar{x}$ varies over regions of order $\left|\bar{a}_{1}\right|$ or $\left|\bar{a}_{2}\right|$ (say, of order $1 \AA$ ); (iii) equation (5.2.5) where both 
integers $s_{1}, s_{2}$ cannot vanish simultaneously (that is, $V_{1,2}$ oscillates quickly in regions where $J_{M \mid}$ is practically constant). Then one concludes that by destructive interference, $\left(U_{\alpha^{\prime}}^{(0)}, V_{1,2} U_{\alpha^{\prime}}^{(0)}\right)_{1}$ has to be very small or negligible. Notice that the argument and the corresponding conclusion do not apply to $\left(U_{\alpha^{\prime}}^{(0)}, V_{1,1} U_{\alpha^{\prime \prime}}^{(0)}\right)_{1}$, as the crucial

$$
\exp \left[\mathrm{i}\left(\sum_{j=1}^{2} s_{j} \boldsymbol{\tau}_{j}\right) \boldsymbol{x}\right]
$$

with $\left(s_{1}, s_{2}\right) \neq(0,0)$, is now lacking.

This argument suggests that, as far as the neutron propagation modes are concerned, $V(\boldsymbol{x})$ inside the fibre as given by the right-hand side of equation (5.1.1) can be approximated (for any shape of the cross-section $\Omega$ ), by $V_{0}(x)+V_{1.1}(z)$, that is by

$$
V(\boldsymbol{x})=\frac{2 \pi \hbar^{2} b}{m} \cdot \frac{1}{\left|\boldsymbol{a}_{1} \times \boldsymbol{a}_{2}\right|} \sum_{l_{3}=-\infty}^{+\infty} \sum_{d_{3}} \delta\left[z-\left(l_{3}+d_{3}\right)\left|\boldsymbol{a}_{3}\right|\right]
$$

which is independent of $\bar{x}$ and of the Krönig-Penney type (Krönig and Penney 1931) regarding the $z$ dependence. We are also assuming that the total number of different $d_{3}$ values equals $n(\{\boldsymbol{d}\})$, which is valid in the cases of interest for us.

Following the above argument, it is natural to accept also that any function $U_{\alpha}(\bar{x}, z)$ characterising a neutron propagation mode in the infinite periodic fibre corresponding to equation (5.1.1) is practically constant as $\overline{\boldsymbol{x}}$ varies over distances of order $\left|\overline{\boldsymbol{a}}_{1}\right|,\left|\overline{\boldsymbol{a}}_{2}\right|$, for fixed $z$ and any shape of the cross-section $\Omega$. Then, based solely upon the last property and as an additional check of consistency, we outline another justification that equation (5.1.1) can be approximated by equation (5.2.9). For this purpose, notice first that for any $V(x)$ which vanishes outside the fibre and is periodic in $z$ with period $\left|\boldsymbol{a}_{3}\right|$, the function $U_{\alpha}(\overline{\boldsymbol{x}}, z)$ satisfies the integral equation

$U_{\alpha}(\overline{\boldsymbol{x}}, z)=-\int_{-a_{3} / 2}^{a_{3}^{\prime} / 2} \mathrm{~d} z^{\prime} \int_{\Omega} \mathrm{d}^{2} x^{\prime} G_{1}\left(\overline{\boldsymbol{x}}-\overline{\boldsymbol{x}}^{\prime} ; z-z^{\prime}\right) \frac{2 m}{\hbar^{2}} V\left(x^{\prime}\right) U_{d}\left(\overline{\boldsymbol{x}}^{\prime}, z^{\prime}\right)$

where the Green's function

$$
\begin{aligned}
G_{1}(\bar{x} ; z)=\frac{1}{\left|a_{3}\right|} & \sum_{n=-\infty}^{-\infty} \int \frac{\mathrm{d}^{2} \bar{l}}{(2 \pi)^{2}} \\
& \quad \times \exp \left[\mathrm{i}\left(\bar{l} \cdot \bar{x}+\frac{2 \pi}{\left|a_{3}\right|} n z\right)\right]\left[\bar{l}+\left(\beta+\frac{2 \pi}{\left|a_{3}\right|} n\right)^{2}-\frac{2 m E}{\hbar^{2}}-\mathrm{i} \varepsilon\right]^{-1}
\end{aligned}
$$

fulfills

$$
\left[-\Delta_{\bar{x}}-\left(\frac{\partial}{\partial z}+\mathrm{i} \beta\right)^{2}-\frac{2 m E}{\hbar^{2}}\right] G_{1}(\bar{x} ; z)=\delta^{(2)}(\bar{x}) \delta(z) .
$$

Since $G_{1}\left(\bar{x}-\bar{x}^{\prime} ; z-z^{\prime}\right)$ is periodic in $z$ with period $\left|a_{3}\right|$ (for fixed $z^{\prime}$ ), the same is true for $U_{a}(\bar{x}, z)$, as given by the right-hand side of equation $(5.2 .10)$. Equations $(5.2 .10-11)$ generalise equations $(\mathrm{A} 2.4-5)$ respectively.

Next, by replacing $V\left(x^{\prime}\right)$ in equation $(5.2 .10)$ by the right-hand side of equation (5.1.1) one finds

$$
\begin{aligned}
U_{\alpha}(\overline{\boldsymbol{x}}, z)=- & 4 \pi b \sum_{i_{1 . l_{2}}} \sum_{d} G_{1}\left(\overline{\boldsymbol{x}}-\sum_{i=1}^{2}\left(l_{i}+d_{i}\right) \overline{\boldsymbol{a}}_{i}, z-d_{3}\left|\boldsymbol{a}_{3}\right|\right) \\
& \times U_{\alpha}\left(\sum_{i=1}^{2}\left(l_{i}+d_{i}\right) \overline{\boldsymbol{a}}_{i}, d_{3}\left|\boldsymbol{a}_{3}\right|\right) .
\end{aligned}
$$


The restriction of equation (5.2.13) to

$$
\overline{\boldsymbol{x}}=\sum_{i=1}^{2}\left(l_{i}^{\prime}+d_{i}\right) \overline{\boldsymbol{a}}_{i} \quad z=d_{3}^{\prime}\left|\boldsymbol{a}_{3}\right|
$$

would yield a homogeneous system of algebraic equations for the large number of amplitudes

$$
U_{\alpha}\left(\sum_{i=1}^{2}\left(l_{i}+d_{i}\right) \bar{a}_{i}, d_{3}\left|a_{3}\right|\right),
$$

and the vanishing of its determinant would give the dispersion relation for the propagation modes, but we shall not pursue this further. Instead, if $U_{\alpha}$ is practically constant as $\overline{\boldsymbol{x}}$ varies over distances of order $\left|\overline{\boldsymbol{a}}_{1}\right|,\left|\overline{\boldsymbol{a}}_{2}\right|$, one may regard $\overline{\boldsymbol{a}}_{1}, \bar{a}_{2}$ and $\left|\boldsymbol{a}_{1} \times \boldsymbol{a}_{2}\right|$ as very small quantities and, so

and

$$
\sum_{i=1}^{2}\left(l_{i}+d_{i}\right) \vec{a}_{i}
$$

$$
\sum_{i_{1}, l_{2}} \sum_{d_{1}, d_{2}}
$$

can be replaced approximately by $\overline{\boldsymbol{x}},\left|\boldsymbol{a}_{1} \times \boldsymbol{a}_{2}\right|^{-1} \cdot \int_{\Omega} \mathrm{d}^{2} \tilde{\boldsymbol{x}}^{\prime}$, respectively. Then equation (5.2.13) can be approximated by equation $(5.2 .10)$, where $V\left(x^{\prime}\right)$ now stands for the right-hand side of equation (5.2.9). In other words, this consistency condition provides additional support for the validity of equation (5.2.9) as the approximate potential seen by a neutron in the infinite periodic fibre.

\subsection{Approximate determination of wave-functions and energies for the potential (5.2.9)}

We obtain the propagation modes and their dispersion relation approximately, when $V(x)$ is given in equation (5.2.9) and the fibre cross-section $\Omega$ is circular (radius $R$ ). The former turn out to be equal, approximately, to those for the homogeneous fibre (see § 3.2), except for $\beta\left|a_{3}\right| \simeq S_{3} \pi\left(S_{3}\right.$ being an integer), where some small forbidden gaps for the neutron energy occur, as could be expected from the periodicity of $V(x)$ along the $z$ direction.

We choose the following factorised form for the neutron wave-function inside the infinite periodic fibre $(|\overline{\boldsymbol{x}}|<R)$ :

$$
\psi(\boldsymbol{x})=\varphi(\bar{x}) \cdot w(z) .
$$

We shall disregard $\varphi(\bar{x})$ and, by assumption, $w(z)$ will satisfy the following onedimensional Schrödinger equation with eigenvalue $E_{z}$ :

$\left(-\frac{\hbar^{2}}{2 m} \frac{\mathrm{d}^{2}}{\mathrm{~d} z^{2}}+\frac{2 \pi \hbar^{2} b}{m\left|\boldsymbol{a}_{1} \times \boldsymbol{a}_{2}\right|} \sum_{l_{3}=-\infty}^{+\infty} \sum_{d_{3}} \delta\left[z-\left(l_{3}+d_{3}\right)\left|\boldsymbol{a}_{3}\right|\right]-E_{z}\right) w(z)=0$

and bear the Bloch form $w(z)=\exp (\mathrm{i} \beta z) w_{1}(z)$, where $w_{1}(z)$ is periodic in $z$, with Period $\left|a_{3}\right|$. We shall distinguish two cases:

5.3.1. Let $\beta$ be such that $\beta\left|a_{3}\right| \neq S_{3} \pi, S_{3}$ being any integer, even if $\beta$ is of order $\AA^{-1}$.

Now, the key property is that $|b|$, as table 1 shows, is quite small compared to 
$\left|\boldsymbol{a}_{3}\right|(\sim 1 \AA)$ so that the potential in equation (5.3.2) amounts to a small perturbation. Then one may apply standard lowest-order perturbation theory. Alternatively, one may suitably extend the method in Kronig and Penney (1931): for instance, one may first set $d_{3}=0$ in equation (5.3.2), use their procedure directly, then generalise it when $d_{3}$ takes on a few values.

The final results read

$$
E_{z} \simeq \frac{\hbar^{2} \beta^{2}}{2 m}+\frac{2 \pi \hbar^{2} b n(\{d\})}{m\left|\boldsymbol{a}_{1} \times \boldsymbol{a}_{2}\right|\left|\boldsymbol{a}_{3}\right|} \quad w(z) \simeq \exp (\mathrm{i} \beta z) .
$$

As a consistency check, notice that $4 \pi b n(\{d\}) /\left|a_{1} \times a_{2}\right|\left|a_{3}\right| \beta^{2}$ is of order $10^{-3}$.

Next, by replacing $\psi(x)$ in equation (2.1.1) for $|\bar{x}|<R$ by the right-hand side of equation (5.3.1), where $w(z), E_{z}$ fulfil equations $(5.3 .2-3)$, one finds

$$
\left(-\frac{\hbar^{2}}{2 m} \Delta_{\dot{\boldsymbol{x}}}+\frac{2 \pi \hbar^{2} b n(\{\boldsymbol{d}\})}{m\left|\boldsymbol{a}_{1} \times \boldsymbol{a}_{2}\right|\left|\boldsymbol{a}_{3}\right|}\right) \varphi(\boldsymbol{x}) \simeq\left(E-\frac{\hbar^{2} \beta^{2}}{2 m}\right) \varphi(\overline{\boldsymbol{x}}) \text {. }
$$

For $|\overline{\boldsymbol{x}}|>R$, one takes $\psi(\boldsymbol{x})=\exp (\mathrm{i} \beta z) \varphi(\overline{\boldsymbol{x}})$, where $\varphi(\overline{\boldsymbol{x}})$ is given in equation (3.2.2). The comparison between equations (5.1.2), (5.3.1), (3.1.1) and the use of (5.3.3-4) shows that approximate consistency is achieved provided that: (i) $\varphi(\bar{x})$ and $E-\left(\hbar^{2} \beta^{2} / 2 m\right)$ in equation (5.3.4) equal the right-hand side of equation (3.2.1) and $-\left(\hbar^{2} \kappa^{2} / 2 m\right)$, respectively; (ii) equations (3.2.3-4) hold, with $\rho=$ $\left(\left|a_{1} \times a_{2}\right|\left|a_{3}\right|^{-1} n(\{d\})\right.$.

5.3.2. Let $\beta$ be very close to $S_{3} \pi /\left|a_{3}\right|, S_{3}$ being an integer (e.g. $\left(\beta-S_{3} \pi\left|a_{3}\right|^{-1 / \beta}\right)<$ $\left.10^{-3}\right)$.

To be definite, let $S_{3}$ be positive. Then, there are forbidden band gaps for $E_{z}$. By using the proper restriction of equation (A1.8) for $\Lambda=0$ and for the $z$ axis, the standard application of Brillouin-Wigner perturbation theory ( $\$ 3.2$ in Ziman 1972) yields

$$
\begin{aligned}
& E_{z_{1} \mp}\left(S_{3}\right) \simeq \frac{\hbar^{2}}{2 m}\left(\frac{S_{3} \pi}{\boldsymbol{a}_{3} \mid}\right)^{2} \mp\left|\tilde{V}\left(S_{3}\right)\right| \\
& \hat{V}\left(S_{3}\right)=\frac{2 \pi \hbar^{2} b}{m\left|\boldsymbol{a}_{1} \times \boldsymbol{a}_{2}\right|\left|\boldsymbol{a}_{3}\right|} \sum_{\boldsymbol{d}} \exp \left(-\mathrm{i} S_{3} \boldsymbol{\tau}_{3} d\right) \\
& W_{\mp}(z) \simeq\left\{\begin{array}{l}
\cos \left(S_{3} \pi /\left|\boldsymbol{a}_{3}\right|\right) z \\
\sin \left(S_{3} \pi /\left|\boldsymbol{a}_{3}\right|\right) \mathrm{z} .
\end{array}\right.
\end{aligned}
$$

Thus, one finds the sequence of forbidden bands $E_{z,-}\left(S_{3}\right)<E_{z}<E_{z,+}\left(S_{3}\right)$, as $S_{3}$ varies, having widths $2\left|\tilde{V}\left(S_{3}\right)\right|$, where no neutron propagation modes exist.

Near the upper (lower) edge of an allowed band, where $E_{z} \leqslant E_{z,-}\left(S_{3}\right)$ $\left(E_{z} \geqslant E_{z,+}\left(S_{3}\right)\right), \psi_{+}(x)$ in equation (5.3.1) for $|\bar{x}|<R$ equals $w_{\mp}(z) \varphi=(\bar{x})$, where

$$
\left(-\frac{\hbar^{2}}{2 m} \Delta_{\tilde{x}} \mp\left|\bar{V}\left(S_{3}\right)\right|\right) \varphi_{\mp}(\overline{\boldsymbol{x}})=\left[E-\frac{\hbar^{2}}{2 m}\left(\frac{S_{3} \pi}{\left|\boldsymbol{a}_{3}\right|}\right)^{2}\right] \varphi_{\mp}(\overline{\boldsymbol{x}}) .
$$

Consequently, for the upper edge, one has an attractive potential in equation (5.3.8), $\varphi_{-}(\overline{\boldsymbol{x}})$ is given by the right-hand side of equation (3.2.1) and

$$
E-\frac{\hbar^{2}}{2 m}\left(\frac{S_{3} \pi}{\left|a_{3}\right|}\right)^{2}=-\frac{\hbar^{2} \kappa^{2}}{2 m} \text {. }
$$


For $|\overline{\boldsymbol{x}}|>R, \psi_{-}(\overline{\boldsymbol{x}})$ equals $w_{-}(z)$ times the right-hand side of equation (3.2.2), and equations (3.2.3-4) still hold, provided that $\rho$ be replaced by

$$
\left(\left|a_{1} \times a_{2}\right|\left|a_{3}\right|\right)^{-1}\left[\sum_{d} \exp \left(-\mathrm{i} S_{3} \tau_{3} d\right)\right] .
$$

Since $w_{-}(z)$ (equation (5.3.7a)) is almost a standing wave (due to Bragg reflections) instead of a progressive one, the net flux of neutrons along the fibre should be extremely small at the upper edge $\left(E_{z} \leqslant E_{z_{1}-}\left(S_{3}\right)\right)$.

On the other hand, for the lower edge, equation (5.3.8) contains a repulsive potential and hence it has no bound states! The overall consequence is that there must exist some $E_{z}\left(S_{3}\right)^{\prime}$ inside the allowed band above $E_{z,+}\left(S_{3}\right)$ and quite close to the latter, so that there are no neutron propagation modes in the small interval $E_{z .+}\left(S_{3}\right)<E_{z}<E_{z}\left(S_{3}\right)^{\prime}$, although they do exist in the relatively large interval $E_{z}\left(S_{3}\right)^{\prime}<E_{z}<E_{z,-}\left(S_{3}+1\right)$.

It is possible to extend to the actual periodic fibre the methods and results outlined in $\S 4$ and appendix 2 for the homogeneous one. Here we omit them for the sake of brevity. Also, the propagation modes for the periodic fibre when $\beta\left|a_{3}\right| \neq S_{3} \pi$ ( $S$ being an integer) reduce as a first approximation to those of the homogeneous fibre, so that the analysis in $\S 4$ and appendix 2 could be applied.

\section{Acknowledgments}

We are grateful to $\mathrm{M}$ Chevalier and to Drs V Alcober and O Schaerpf for useful discussions. The partial financial support given by Comisión Asesora de Investigación Científica y Técnica, Spain, is also acknowledged.

\section{Appendix 1}

Here we construct a physically acceptable approximate expression for the potential seen by a confined neutron in an infinite periodic crystal lattice, whose atoms vibrate at absolute temperature $T$. We consider an unmagnetised material and $\operatorname{Re} b<0, \operatorname{Im} b=$ 0 . We follow the notations in $\$ 2.1$ of Marshall and Lovesey (1971). The basic lattice vectors are $a_{1}, a_{2}, a_{3}$. If the lattice were rigid, its atoms would be at the positions $l+d$, where $l=\sum_{i=1}^{3} l_{i} a_{i}$ ( $l_{i}$ being integers taking on all possible values) and $d=$ $\sum_{i=1}^{3} d_{i} a_{i}\left(d_{i}\right.$ being certain numbers with the restriction $\left.0 \leqslant d_{i} \leqslant 1\right)$.

As in chapter 1 of Marshall and Lovesey (1971) and in Greenberger and Overhauser (1979), we take the (coherent) potential seen by the neutron due to the infinite rigid lattice as given by the standard sum of Fermi pseudopotentials:

$$
\begin{aligned}
\frac{2 \pi \hbar^{2} b}{m} \sum_{l, d} \delta^{(3)} & {[x-(l+d)] } \\
= & \frac{\hbar^{2} b}{m(2 \pi)^{2}} \sum_{l, d} \int \mathrm{d}^{3} q \exp \{i q[x-(l+d)]\} \equiv V[x,\{l+d\}]
\end{aligned}
$$

where the summations run over all possible $l, d$. Equation (A1.1), which is our starting point, turns out to be (approximately) the final result for the potential due to the vibrating lattice at ordinary temperatures.

For a vibrating lattice, one should replace each $l+d$ by $l+d+v(l+d)$, where 
$\boldsymbol{v}(\boldsymbol{l}+\boldsymbol{d})$ is the (small) displacement of the atom at $\boldsymbol{l}+\boldsymbol{d}$, which is the standard linear combination of phonon, creation and absorption operators (see $\S 4.1$ in Marshall and Lovesey 1971).

Let $V\{\boldsymbol{x},[\boldsymbol{l}+\boldsymbol{d}+\boldsymbol{v}(\boldsymbol{l}+\boldsymbol{d})]\}$ be the operator which results after performing the above replacement in equation (A1.1) and let $H_{\mathrm{ph}}$ be the usual phonon hamiltonian for the harmonic vibrations of the lattice. Then

$$
\begin{aligned}
& \frac{p^{2}}{2 m}+H_{\mathrm{ph}}+V\{\boldsymbol{x},[\boldsymbol{l}+\boldsymbol{d}+\boldsymbol{v}(\boldsymbol{l}+\boldsymbol{d})]\}-H_{1,0} \equiv H_{1} \\
& H_{1,0}=\left\{\operatorname{Tr}_{\mathrm{ph}}\left[\exp \left(-\frac{H_{\mathrm{ph}}}{K_{\mathrm{B}} T}\right)\right]\right\}^{-1} \operatorname{Tr}_{\mathrm{ph}}\left[H_{\mathrm{ph}} \exp \left(-\frac{H_{\mathrm{ph}}}{K_{\mathrm{B}} T}\right)\right]
\end{aligned}
$$

can be regarded as the hamiltonian for the vibrating harmonic lattice interacting with one neutron, $K_{\mathrm{B}}$ being Boltzmann's constant. Notice that: (i) $H_{1}$ acts in the product space of the phonon Fock space times the space containing the neutron wave functions, (ii) for any operator $A: \operatorname{Tr}_{\mathrm{ph}} A=\Sigma_{\text {all }}\langle\xi|A| \xi\rangle$, where $\xi$ is any normalised vibrational state of the lattice, containing a definite number of phonons (with given wave-vectors and polarisation indices), which is an eigenstate of $H_{\mathrm{ph}}$ with eigenvalue $E(\xi)$; (iii) we have carried out, for later convenience, a finite renormalisation consisting in the subtraction of the lattice energy at temperature $T$, namely, $H_{1.0}$ (equation (A1.3)).

The variational principle implies that the exact ground-state energy of $H_{1}$ is smaller than (or equal to) $\left\langle\psi \otimes \xi\left|H_{1}\right| \xi \otimes \psi\right\rangle$, where $\xi$ is the same genetic vibrational state of the lattice as before and $\psi$ is an arbitrary normalised wave function for the neutron. Consequently, by keeping $\psi$ fixed, one sees easily that the exact ground-state energy of $H_{1}$ is also smaller than (or equal to)

$$
\begin{aligned}
\left\{\operatorname{Tr}_{\mathrm{ph}}\left[\exp \left(-\frac{H_{\mathrm{ph}}}{K_{\mathrm{B}} T}\right)\right]\right\}^{-1} \sum_{\mathrm{all}}\left\langle\psi \otimes \xi\left|H_{1}\right| \xi \otimes \psi\right\rangle \exp \left(-\frac{E(\xi)}{K_{\mathrm{B}} T}\right) \\
=\left\{\operatorname{Tr}_{\mathrm{ph}}\left[\exp \left(-\frac{H_{\mathrm{ph}}}{K_{\mathrm{B}} T}\right)\right]\right\}^{-1}\left\langle\psi\left|\operatorname{Tr}_{\mathrm{ph}}\left[H_{1} \exp \left(-\frac{H_{\mathrm{ph}}}{K_{\mathrm{B}} T}\right)\right]\right| \psi\right\rangle
\end{aligned}
$$

By following the arguments in $\S 3.7$ in Marshall and Lovesey (1971) it is easy to show that

$$
\begin{array}{r}
\left\{\operatorname{Tr}_{\mathrm{ph}}\left[\exp \left(-\frac{H_{\mathrm{ph}}}{K_{\mathrm{B}} T}\right)\right]\right\}^{-1} \operatorname{Tr}_{\mathrm{ph}}\left[\mathrm{V}\{\boldsymbol{x},[\bar{l}+\boldsymbol{d}+\boldsymbol{v}(\boldsymbol{l}+\boldsymbol{d})]\} \exp \left(-\frac{H_{\mathrm{ph}}}{K_{\mathrm{B}} T}\right)\right] \\
=\frac{\hbar^{2} b}{m(2 \pi)^{2}} \sum \int \mathrm{d}^{3} \boldsymbol{q} \exp \{\mathbf{i} \boldsymbol{q}[\boldsymbol{x}-(\boldsymbol{l}+\boldsymbol{d})]\} \exp \left[-W_{d}(\boldsymbol{q})\right] \equiv V_{v}(\boldsymbol{x})
\end{array}
$$

where

$$
W_{d}(\boldsymbol{q})=\frac{\hbar\left[\left(\boldsymbol{a}_{1} \times \boldsymbol{a}_{2}\right) \boldsymbol{a}_{3}\right]}{4 M(2 \pi)^{3}} \int \mathrm{d}^{3} \boldsymbol{q}^{\prime} \sum_{j} \frac{\left[\left.\boldsymbol{q} \cdot \boldsymbol{\sigma}_{d}^{i}\left(\boldsymbol{q}^{\prime}\right]\right|^{2}\right.}{\omega_{j}\left(\boldsymbol{q}^{\prime}\right)}\left\{1+2\left[\exp \left(\frac{\hbar \omega_{j}\left(\boldsymbol{q}^{\prime}\right)}{K_{\mathrm{B}} T}\right)-1\right]^{-1}\right\}
$$

$M$ is the atomic mass, and $\omega_{j}\left(q^{\prime}\right), \boldsymbol{\sigma}_{d}^{j}$ are the frequency and a possible polarisation vector for a phonon having wave-vector $q^{\prime}$. The summation and integration on the right-hand side of equation (A1.6) extend over all allowed $j$ and $\boldsymbol{q}^{\prime}$. Notice that $\exp \left[-W_{d}(q)\right]$ is the standard Debye-Waller factor and that the above variational argument for the interaction of a neutron with phonons in an infinite vibrating crystal has led us to the same potential which is commonly used in the analysis of elastic 
coherent scattering of neutrons by crystals ( $\$ 4.2$ in Marshall and Lovesey (1971), Greenberger and Overhauser (1979), and references therein).

Hence, the exact ground-state energy of $H_{1}$ is less than or equal to

$$
\left\langle\psi\left|\left[\frac{\boldsymbol{p}^{2}}{2 m}+V_{v}(\boldsymbol{x})\right]\right| \psi\right\rangle
$$

for any normalised neutron wave-function $\psi$. A posteriori, we can appreciate that in order to arrive at the above result, the finite renormalisation $-H_{1.0}$ was essential.

The spirit of the above argument should not be misunderstood. It is well known (see for instance Lax (1951)) that the incident energy of an incoming thermal neutron which interacts with a vibrating crystal can be very different from that of the scattered one, due to phonon excitation. In this appendix, we are not concerned with neutron scattering, but rather with the stationary states that a neutron could have in an infinite crystal occupying all space, to which the variational principle applies. Our physical approximation consists in the replacement of the complicated neutron-phonon interaction by the potential $V_{v}$ regarding the description of any stationary state for the neutron in the infinite crystal.

We next obtain some approximate expressions for equations (A1.5-6) at normal temperatures (say, $T=300 \mathrm{~K}$ ) for most of the elements listed in table 1 .

Let us assume: (i) a Bravais lattice structure, which is true for $\mathrm{Li}$ (bcc structure). $\mathrm{V}$ (bcc). Mn (sc) Ni (fcc), so that $d=0$ and, (ii) a typical Debye spectrum for the phonon frequencies (see $\$ 4.2$ in Marshall and Lovesey 1971). Then, according to Marshall and Lovesey (1971) the high-temperature expression:

$$
W(q)=\frac{1}{2} \Lambda^{2} \bar{q}^{2} \quad \Lambda^{2}=\frac{3 \hbar^{2}}{M} \frac{1}{K_{\mathrm{B}} \theta_{\mathrm{D}}} \frac{T}{\theta_{\mathrm{D}}}
$$

is approximately valid for $T \geqslant 0.4 \theta_{\mathrm{D}}$. The last inequality holds for $T=300 \mathrm{~K}$ and for the Debye temperatures $\theta_{\mathrm{D}}$ of $\mathrm{Li}, \mathrm{Ti}, \mathrm{Mn}, \mathrm{Ni}$ and $\mathrm{Sm}$ (see Kittel 1966, p 180, and Gschneider 1964).

We note that the $\mathrm{Ti}$ lattice is an hcp structure with two atoms per unit cell, corresponding to $d=0$ and

$$
d=\frac{1}{3}\left(a_{1}+a_{2}\right)+\frac{1}{2} a_{3}
$$

(see $\$ 2.1$ in Marshall and Lovesey 1971) but not a Bravais lattice. Then the above simple expressions (A1.7) may not be justified a priori for Ti. A detailed calculation of $W_{j}$ for $\mathrm{Ti}$ would require the previous evaluation of the corresponding phonon frequencies and polarisation vectors, a task which lies far outside the scope of the present work. However, one may argue that since $\omega_{j}\left(\boldsymbol{q}^{\prime}\right)$ and $\boldsymbol{\sigma}_{d}^{j}\left(\boldsymbol{q}^{\prime}\right)$ are integrated over in order to obtain $W_{j}$, their explicit forms may not be so important: in particular, the angular integration in equation (A1.6) should tend to smooth out the peculiarities of the $\boldsymbol{\sigma}_{a}^{l}$ corresponding to an hcp structure. Then one may tentatively use equations (A1.7) for Ti as well, with its associated Debye temperature $\theta_{\mathrm{D}}$ : the order of magnitude and the main features for the resulting $V_{v}(\boldsymbol{x})$ should not be altered drastically by the above approximation.

Consequently, by using the approximation (A1.7) in equation (A1.5), performing the gaussian integration over $q$ and, then, applying the Poisson sum formula (see $\$ 2.3$ in Ziman 1972), one finds successively the following approximate expressions for 
$V_{v}(\boldsymbol{x})$

$$
\begin{aligned}
& V_{v}(\boldsymbol{x}) \simeq \frac{\hbar^{2} b}{m(2 \pi)^{1 / 2} \Lambda^{3}} \sum_{l, d} \exp \left(-\frac{1}{2 \Lambda^{2}}[\boldsymbol{x}-(\boldsymbol{l}+\boldsymbol{d})]^{2}\right) \\
& =\frac{\hbar^{2} b 2 \pi}{m\left[\left(\boldsymbol{a}_{1} \times \boldsymbol{a}_{2}\right) \boldsymbol{a}_{3}\right]} \sum_{\tau} \exp (\mathrm{i} \boldsymbol{\tau} \boldsymbol{x})\left(\sum_{\boldsymbol{d}} \exp (-\mathrm{i} \boldsymbol{\tau} \boldsymbol{d})\right) \exp \left(-\frac{\Lambda^{2} \boldsymbol{\tau}^{2}}{2}\right) \\
& \boldsymbol{\tau}=\sum_{i=1}^{3} s_{i} \boldsymbol{\tau}_{i} \quad \boldsymbol{\tau}_{i}=\frac{2 \pi}{\left(\boldsymbol{a}_{1} \times \boldsymbol{a}_{2}\right) \boldsymbol{a}_{3}} \boldsymbol{a}_{j} \times \boldsymbol{a}_{k}
\end{aligned}
$$

$s_{i}$ being arbitrary integers and $(i, j, k)$ being a cyclic permutation.

We shall assess the magnitude of $\Lambda^{2} \tau^{2} / 2$. Using the first equation (A1.7), the second equation (A1.9) and the explicit expressions for $\tau_{1}, \tau_{2}, \tau_{3}$ given in $\S 2.1$ of Marshall and Lovesey (1971), one gets for $\mathrm{Li}, \mathrm{V}, \mathrm{Mn}$ and ${ }^{62} \mathrm{Ni}$ :

$$
\frac{\Lambda^{2} \tau^{2}}{2}=\left(\sum_{i=1}^{3} s_{i}^{2}+\xi\left(s_{1} s_{2}+s_{2} s_{3}+s_{3} s_{1}\right)\right) y
$$

and for Ti:

$$
\begin{aligned}
& \frac{\Lambda^{2} \boldsymbol{\tau}^{2}}{2}=\left(s_{1}^{2}+s_{2}^{2}+0.29 s_{3}^{2}+\xi s_{1} s_{2}\right) y \\
& y=y_{0} \frac{2 \pi^{2}}{a^{2}} \Lambda^{2} .
\end{aligned}
$$

We give in table 4 the explicit values of $\xi, y_{0}, a, y$ and $\Lambda$ for the above elements. The values of the unit cell parameter $a$ for $\mathrm{Li}, \mathrm{Ti}, \mathrm{V}$ and $\mathrm{Ni}$ were taken directly from Kittel (1966, p 26), while that for Mn was calculated from its atomic weight and density. We used the specific values of $\theta_{\mathrm{D}}$ for $\mathrm{Li}$, and Ti given in Kittel (1966, p 180), and estimated those for V, Mn and Ni from the data in Gschneider (1964). Notice that the (average) values of $a, \theta_{\mathrm{D}}$ for $\mathrm{Ni}$ have been taken as identical to those for ${ }^{62} \mathrm{Ni}$. Finally, by looking at the values for $y, y_{0}, a$, and $\Lambda$ displayed in table 4 , one sees that the replacement

Table 4. Unit cell parameters.

\begin{tabular}{llllll}
\hline & \multicolumn{5}{c}{ Element } \\
\cline { 2 - 6 } & $\mathrm{Li}$ & $\mathrm{Ti}$ & $\mathrm{V}$ & $\mathrm{Mn}$ & ${ }^{62} \mathrm{Ni}$ \\
\hline$\xi$ & $\frac{1}{2}$ & -1 & $\frac{1}{2}$ & 0 & $-\frac{2}{3}$ \\
$y_{0}$ & 2 & $\frac{1}{3}$ & 2 & 1 & 3 \\
$a(\AA)$ & 3.5 & 2.95 & 3 & 2.33 & 3.5 \\
$y$ & 0.18 & 0.015 & 0.03 & 0.02 & 0.02 \\
$\Lambda(\overline{\mathrm{A}})$ & 0.24 & 0.07 & 0.08 & 0.07 & 0.06 \\
\hline
\end{tabular}

of $\left[(2 \pi)^{3 / 2} \Lambda^{3}\right]^{-1} \exp \left\{-\left(1 / 2 \Lambda^{2}\right)\left[(x-(l+d)]^{2}\right\}\right.$ by $\delta^{(3)}[x-(l+d)]$ is approximately justified (since $\left[(2 \pi)^{3 / 2} \Lambda^{3}\right]^{-1} \exp \left\{-\left(1 / 2 \Lambda^{2}\right)\left[(x-(l+d)]^{2}\right\} \rightarrow \delta^{(3)}[x-(l+d)]\right.$ if $\left.\Lambda \rightarrow 0\right)$. All this justifies, a posteriori, that at oruinary temperatures $(300 \mathrm{~K}) V_{v}(\boldsymbol{x})$ can be replaced, as a first approximation, by the rigid lattice potential $V[x,(l+d)]$ (equation (A1.1)). 


\section{Appendix 2. The penetration of an incoming neutron wave into a fibre: an integral equation description}

We consider the process through which an incoming free-neutron wave $\psi_{\text {in }}(\boldsymbol{x})$, satisfying equation (2.1.1) with $E>0$ and $V(x)=0$ for any $x$, penetrates into the cylindrical fibre (located in $0<z<z_{0}$ ) and propagates along it. Here, we also allow for nuclear absorption inside the fibre: $\operatorname{Im} b \leqslant 0, \operatorname{Im} V \leqslant 0$.

The starting point is the following standard three-dimensional scattering integral equation satisfied by the total wave-function of the neutron for any $x=(\bar{x}, z)\left(x^{\prime}=\right.$ $\left.\left(\overline{\boldsymbol{x}}^{\prime}, z^{\prime}\right), \varepsilon \rightarrow 0^{+}\right)$:

$$
\begin{aligned}
& \psi(\boldsymbol{x})=\psi_{\text {in }}(\boldsymbol{x})-\int_{\Omega} \mathrm{d}^{2} \boldsymbol{x}^{\prime} \int_{0}^{z_{0}} \mathrm{~d} z^{\prime} G\left(\overline{\boldsymbol{x}}-\overline{\boldsymbol{x}}^{\prime}, z-z^{\prime}\right) V\left(\boldsymbol{x}^{\prime}\right) \psi\left(\boldsymbol{x}^{\prime}\right) \\
& G\left(\overline{\boldsymbol{x}}-\overline{\boldsymbol{x}}^{\prime}, z-z^{\prime}\right)=\int \frac{\mathrm{d}^{2} \bar{l} \mathrm{~d} l_{z}}{(2 \pi)^{3}} \frac{\exp \left\{\mathrm{i}\left[\bar{l}\left(\boldsymbol{x}-\overline{\boldsymbol{x}}^{\prime}\right)+l_{z}\left(z-z^{\prime}\right)\right]\right\}}{\left.\left(\hbar^{2} / 2 m\right)\left(\bar{l}^{2}+l_{z}^{2}\right)\right)-(E+\mathrm{i} \varepsilon)} .
\end{aligned}
$$

Equation (A2.1) is equivalent to equation (2.1.1) together with the incoming boundary condition determined by $\psi_{\text {in }}(\boldsymbol{x})$. Notice that $V(\boldsymbol{x})=V(\overline{\boldsymbol{x}})$.

The problem is to find the most general $\psi_{\text {in }}(\boldsymbol{x})$ such that $\psi(\boldsymbol{x})$ reduces, for $0<$ $z<z_{0}$, to one or, more generally, to a superposition of generalised propagation modes, namely

$$
\psi(x)=\sum_{\alpha, \beta} c_{\alpha \beta} \exp (\mathrm{i} \beta z) \varphi_{\alpha}(\tilde{x}) \quad 0<z<z_{0}
$$

where $c_{\alpha \beta}$ are ( $x$ independent) constants. The sums (or integrals) in equation (A2.3) extend in principle to all values of $\alpha, \beta$ such that $\left(\hbar^{2} / 2 m\right)\left(\beta^{2}-\kappa^{2}\right)=E>0$ holds, and in turn, $\varphi_{\alpha}(\bar{x})$ and the associated $\kappa$ fulfill the actual analogue of equation (3.1.1). At this point, note that the latter is equivalent to the following bound-state integral equation:

$$
\begin{aligned}
& \varphi_{\alpha}(\overline{\boldsymbol{x}})=\int_{\Omega} \mathrm{d}^{2} \overline{\boldsymbol{x}}^{\prime} \frac{1}{4 \mathrm{i}} H_{0}^{(1)}\left(\mathrm{i} \kappa \mid \overline{\boldsymbol{x}}-\boldsymbol{x}^{\prime}\right)\left[-\frac{2 m}{\hbar^{2}} V\left(\boldsymbol{x}^{\prime}\right)\right] \varphi_{\alpha}\left(\overline{\boldsymbol{x}}^{\prime}\right) \\
& \frac{1}{4 \mathrm{i}} H_{0}^{(1)}\left(\mathrm{i} \kappa\left|\overline{\boldsymbol{x}}-\overline{\boldsymbol{x}}^{\prime}\right|\right)=\int \frac{\mathrm{d}^{2} \bar{l}}{(2 \pi)^{2}} \frac{\exp \left[\mathrm{i} l\left(\boldsymbol{x}-\overline{\boldsymbol{x}}^{\prime}\right)\right]}{\bar{l}^{2}+\boldsymbol{K}^{2}}
\end{aligned}
$$

$H_{0}^{(1)}$ being Hankel's outgoing function of zero order. Notice that $(4 \mathrm{i})^{-1} H_{0}^{(1)}$ is a Green's function for $\Delta_{\dot{x}}-k^{2}$.

Next, we restrict equation (A2.1) to $0<z<z_{0}$ and perform the following operations in it: (i) we replace $\psi(\bar{x})$ in its left-hand side by the right-hand side of equation (A2.3) and, at the same time, in the latter we substitute $\varphi_{a}(\bar{x})$ by the right-hand side of equation (A2.4); (ii) we replace $\psi\left(x^{\prime}\right)$ in its right-hand side by that of equation (A2.3); (iii) by using the representations (A2.2) and (A2.5), we carry out the integration over $z^{\prime}$. Thus, we arrive at the following representation for $\psi_{\text {in }}(\boldsymbol{x})$ in $0<z<z_{0}$ :

$$
\begin{aligned}
\psi_{\text {in }}(\boldsymbol{x})= & \sum_{\alpha, \beta} c_{\alpha \beta} \int_{\Omega} \mathrm{d}^{2} \overline{\boldsymbol{x}}^{\prime} \frac{2 m}{\hbar^{2}} V\left(\overline{\boldsymbol{x}}^{\prime}\right) \varphi_{\alpha}\left(\overline{\boldsymbol{x}}^{\prime}\right)\left(\int \frac{\mathrm{d}^{2} \bar{l}}{(2 \pi)^{2}} \exp \left[\mathrm{i} \bar{l}\left(\overline{\boldsymbol{x}}-\overline{\boldsymbol{x}}^{\prime}\right)\right] \cdot \Lambda_{\alpha \beta}(z, \bar{l})\right) \\
\Lambda_{\alpha \beta}(z, \bar{l})= & \int_{-\infty}^{+\infty} \frac{\mathrm{d} l_{z}}{2 \pi \mathrm{i}} \frac{\exp \left(\mathrm{i} l_{z} z\right)}{\bar{l}^{2}+l_{z}^{2}-\left(2 m E / \hbar^{2}\right)-\mathrm{i} \varepsilon} \frac{\exp \left[\mathrm{i} z_{0}\left(\beta-l_{z}\right)\right]-1}{\beta-l_{z}} \\
& -\frac{\exp (\mathrm{i} \beta z)}{\tilde{l}^{2}+\kappa^{2}} .
\end{aligned}
$$


By performing a residue integration, one finds

$$
\begin{aligned}
& \Lambda_{\alpha \beta}(z, \bar{l})=\frac{1}{2 \sigma}\left(\frac{\exp (\mathrm{i} \sigma z)}{\sigma-\beta}+\frac{\exp (-\mathrm{i} \sigma z) \exp [\mathrm{i}(\sigma+\beta)] z_{0}}{\sigma+\beta}\right) \\
& \sigma=\left(\frac{2 m E}{\hbar^{2}}-\bar{l}^{2}\right)^{1 / 2} .
\end{aligned}
$$

It is easy to check that $\psi_{\text {in }}(x)$, as given by equations (A2.6), (A2.8-9) does satisfy $\left[-\left(\hbar^{2} / 2 m\right) \Delta-E\right] \psi_{\text {in }}=0$ for $-\infty<z<+\infty$. In other words, equations (A2.6), (A2.8-9) yield $\psi_{\text {in }}(\boldsymbol{x})$ not only for $0<z<z_{0}$ but also throughout all space. We have checked the last result affirmatively by using standard Green's function techniques.

The use of equation (A2.3) and of the second term on the right-hand side of equation (A2.1) for $z<0$ and, if $z_{0}<+\infty$, for $z>z_{0}$, yield respectively, the reflected (or backward-scattered) wave $\psi_{\mathrm{re}}$ and the transmitted (or forward-scattered) one $\psi_{\mathrm{tr}}$, generated by the interaction of $\psi_{\text {in }}$ with the fibre. After further residue integrations both turn out to be given by the right-hand side of equation (A2.6), provided that $\Lambda_{\alpha \beta}$ be replaced by $\Lambda_{\mathrm{re}, \alpha \beta}(z, \bar{l})$ and $\Lambda_{\mathrm{tr}, \alpha \beta}(z, \bar{l})$, respectively. In turn

$$
\left.\Lambda_{j, \alpha \beta}(z, \bar{l})=-\frac{q(j) \exp (\mathrm{i} q(j) \sigma z)}{2 \sigma[\sigma-q(j) \beta]} \llbracket 1-\exp \left\{\mathrm{i} z_{0}[\beta-q(j) \sigma]\right\}\right]
$$

where for $j=$ re, $q(j)=-1$ while if $j=\operatorname{tr}, q(j)=+1(\sigma$ being still given by equation (A2.9)). The explicit expressions for the total wave-function $\psi=\psi_{\text {in }}+\psi_{\text {re }}$ in $z<0$ and $\psi=\psi_{\text {in }}+\psi_{\text {tr }}$ in $z>z_{0}$ can be obtained immediately from the above results.

The previous study has the following physical consequences and applications. Let $\mathscr{H}_{\text {in }}$ be the space formed by all free-neutron wave-functions $\psi_{\text {in }}$, given through equations (A2.6) and (A2.8-9), for any possible choice of $c_{\alpha \beta}$. Let $\psi_{0}=\psi_{0}(x)$ be an arbitrary incoming free-neutron wave-function $\left(\left[-\left(\hbar^{2} / 2 m\right) \Delta-E\right] \psi_{0}=0\right.$ for any $\left.\boldsymbol{x}\right)$ : for instance, $\psi_{0}=\exp (\mathrm{i} k \boldsymbol{x})$. Then, one has the unique decomposition $\psi_{0}=\psi_{\text {in }}+\psi_{\text {in }}^{\prime}$ where $\psi_{\text {in }}$ belongs to $\mathscr{H}_{\text {in }}$ and $\psi_{\text {in }}^{\prime}$ lies outside the latter. That is, $\psi_{\text {in }}^{\prime}$ is orthogonal to all wave-functions inside $\mathscr{H}_{\text {in }}$ while $\left[-\left(\hbar^{2} / 2 m\right) \Delta-E\right] \psi_{\text {in }}^{\prime}=0$ for any $\boldsymbol{x}$. Consequently, the total wave-function $\psi_{\text {tot }}$ generated by the interaction of $\psi_{0}$ with the fibre (which is a solution of equation (A2.1) when $\psi, \psi_{\text {in }}$ are replaced by $\psi_{\text {tot }}, \psi_{0}$ ) can be decomposed uniquely as $\psi_{\text {tot }}=\psi+\psi^{\prime}$. Here, $\psi$ is the superposition of generalised propagation modes given by equation (A2.3) for $0<z<z_{0}$ and generated by $\psi_{\text {in }}$, as described above. On the other hand, $\psi^{\prime}$ solves equation (A2.1) (with $\psi_{\text {in }}, \psi$ replaced by $\left.\psi_{\text {in }}^{\prime}, \psi^{\prime}\right)$ and describes only neutron scattering by the fibre but never generalised propagation modes along the latter. For instance, a plane wave $\left(\psi_{0}=\exp (\mathrm{i} k \boldsymbol{x})\right)$ always gives rise uniquely to a superposition of propagation modes inside the fibre $\left(\psi_{\text {in }} \neq 0, \psi \neq 0\right)$ plus a pure scattering contribution $\left(\psi_{\text {in }}^{\prime} \neq 0, \psi^{\prime}-\psi_{\text {in }}^{\prime} \neq 0\right)$.

The following comments may help to clarify the relevance of the decomposition: $\psi_{0}=\psi_{\text {in }}+\psi_{\text {in }}^{\prime}, \psi_{\text {tot }}=\psi+\psi^{\prime}$. It does not seem easy to obtain $\psi$ (equation (A2.3)), by direct successive iterations of equation (A2.1) when $\psi_{\text {in }}$ is given initially through equations (A2.6) and (A2.8-9), unless delicate cancellations be carried out. Consequently, the determination of $\psi_{\text {to }}$ by successive iterations of equation (A2.1) (with $\psi, \psi_{\text {in }}$ replaced by $\psi_{\text {tot }}, \psi_{0}$ ) does not appear to be straightforward either. However, one may reasonably expect that the analogue of equation (A2.1), with $\psi, \psi$ in substituted by $\psi^{\prime}, \psi_{\text {in }}^{\prime}$, enables one to determine $\psi^{\prime}$ by convergent successive iterations (for small $V$ ) or by some other standard method in scattering theory.

Finally, notice that the neutron current along the waveguide (namely, $\left.(\hbar / m) \operatorname{Re}\left[\psi^{*}(-\mathrm{i} \boldsymbol{\nabla}) \psi\right]\right)$ can be obtained directly from equation (A2.3). 
The main results obtained in appendix 2 are: (i) a general characterisation of those incoming wave-functions $\psi_{\text {in }}$ (equations (A2.6) and (A2.8-9)) which give rise to generalised neutron propagation modes (equation (A2.3)) inside the fibre; (ii) an outline of how an arbitrary incoming wave function $\psi_{0}$ decomposes uniquely into a part $\left(\psi_{\text {in }}\right)$ which produces generalised propagation modes, plus a second contribution ( $\psi_{\text {in }}^{\prime}$ ) orthogonal to $\psi_{\text {in }}$, which gives rise to neutron scattering by the fibre: (iii) an indication of how the total wave $\psi^{\prime}$ generated by $\psi_{\text {in }}^{\prime}$ should be calculated. Those results provide a basis for a description of neutron scattering and confinement by the fibre, in the framework of scattering theory.

\section{References}

Abramowitz M and Stegun I A (eds) 1965 Handbook of Mathematical Functions (New York: Dover) ch 9

Bacon G E 1962 Neutron Diffraction (Oxford: Clarendon)

Carlile C J. Johnson M W and Williams W G 1979 Rutherford Lab. Rep. RL 79-084

Christ J and Springer T 1962 Nukleonik 423

Dederichs P H 1972 Solid State Phys. (Adv. Res. Appl.) 27135

Ekstein H 1953 Phys. Rev. 89490

Fowler J F 1981 Nuclear Particles in Cancer Treatment (Bristol: Adam Hilger)

Goldberger M L and Seitz F 1947 Phys. Rev. 71294

Grant T J and Cobble J W 1969 Phys. Rev. Lett. 23741

Greenberger D M and Overhauser A W 1979 Rev. Mod. Phys. 5143

Gschneider K A Jr 1964 Solid State Physics (Adv. Res. Appl.) 16275

Halpern O. Hamermesh M and Johnson M H 1941 Phys. Rev. 59981

Hofmeyr C and Isebeck K 1974 Nucl. Instrum. Methods 1179

Hughes D J and Burgy M T 1951 Phys. Rev. 81498

Hughes D J 1954 Veutron Optics (New York: Wiley Interscience)

Jacrot B 1970 in Proc. Symp. Instrumentation for Neutron Inelastic Scattering Research (Vienna: IAEA)

Kagan Y 1970 JETP Lett. 11147

Kittel C 1966 Introduction to Solid State Physics 3rd ed. (New York: Wiley)

Klein A G and Werner S A 1983 Rep. Prog. Phys. 46259

Koester L. Rauch H. Herkens M and Schröder K 1981 Summary of Neutron Scattering Lengths Atom-institut der Österreichischen Universitaten Jül 1755

Kronig R de L and Penney W G 1931 Proc. R. Soc. A130 499

Lax M 1951 Rev. Mod. Phys. 23287

- 1952 Phvs. Rev. 85621

Maghabghab S F. Divadeenam M and Holden X E 1981 Neitron Cross Sections Vol 1 (National Nuclear Data Center. Brookhaven National Lab.) (New York: Academic)

Maier-Leibnitz H and Springer T 1963 Reactor Sci. Technol. 17217

Marcuse D 1972 Light Transmission Optics (New York: Van Nostrand Reinhold)

Marshall W and Lovesey S W 1971 Theory of Thermal Neutron Scattering (Oxford: Clarendon)

Martin A 1972 Helv. Phys. Acta 45140

Marx D 1971 Nucl. Instrum. Methods 94533

Olshansky R 1979 Rev. Mod. Phys. 51341

Schaerpf $O$ and Eichler D 1973 J. Phys. E: Sci. Instrum. 6774

Sears V F 1982 Phy's. Rep. 821

Shull C G and Wollan E O 1951 Phys. Rev. 81527

Shull C G, Zeilinger A. Squires G L, Horne M A, Atwood D K and Arthur J 1980 Phys. Rev. Lett. 441715

Sidhu S S. Heaton L, Zauberis D D and Campos F P 1956 J. Appl. Phys. 271040

Slater J C 1958 Rev. Mod. Phys. 30197

Steyerl A 1977 in Springer Tracts in Modern Physics ed. G Höhler vol 180 (New York: Springer) pp 57-125

Tien P K 1977 Rev, Mod. Phys. 49361

Windsor C G 1981 Pulsed Neutron Scattering (London: Taylor and Francis)

Zeilinger A and Shull C G 1979 Phys. Rev. B 193975

Zel'dovich Ya B 1959 Soviet Phys.-JETP 91389

Ziman J H 1972 Principles of the Theory of Solids 2nd Edn (Cambridge: Cambridge University Press) 